\title{
Pricing and Inventory Management
}

\author{
Xin Chen*and David Simchi-Levi ${ }^{\dagger}$
}

October 29,2010

\section{Introduction}

Recent years have witnessed phenomenal growth of successful deployments of innovative pricing strategies in a variety of industries. For instance, no company underscores the impact of the Internet on product pricing strategies more than Dell Computers. The price of a product is not fixed on Dell's Web site; it may change significantly over time. Of course, Dell is not alone in its use of sophisticated pricing strategies. Indeed, scores of retail and manufacturing companies have started exploring dynamic pricing to improve their operations and ultimately their bottom line.

Several factors contribute to the phenomenal growth of dynamic pricing. First, the development of sophisticated information technologies greatly facilitates the collection and communication of customer data. Second, costs associated with changing prices have been significantly reduced again due to the development of information technologies. Third, active academic research provides analytical models and tools for price optimization. Fourth, decision support systems for customer data analysis and price optimization have been developed and successfully implemented in a number of industries.

The purpose of this chapter is to survey academic research on price optimization models in which inventory replenishment plays a critical role. Our emphasis is on integrated produc-

${ }^{*}$ Department of Industrial and Enterprise Systems Engineering, University of Illinois at Urbana-Champaign. Email: xinchen@illinois.edu.

${ }^{\dagger}$ Department of Civil and Environmental Engineering, and the Engineering System Division, MIT. Email: dslevi@mit.edu. 
tion/inventory and pricing models that have the potential to be used for decision support at both the operational and the tactical levels. We also review strategic models on supply chain competition, coordination and cooperation built upon these operational and tactical inventory and pricing models.

This chapter is not the first survey of this kind. In fact, several notable survey articles appeared since the publication of the seminal paper by Whitin (1955) who analyzes an EOQ model and a newsvendor model both with price dependent demand. Among them, Eliashberg \& Steinberg (1991) review the literature up to year 1991 on the interface of operations and marketing with an emphasis on integrated inventory and pricing models. Three recent papers, Elmaghraby \& Keskinocak (2003), Yano \& Gilbert (2003) and Chan et al. (2004), survey the related literature up to year 2004 from operations research and management science perspective. Elmaghraby \& Keskinocak (2003) focus on a few key papers on dynamic pricing in the presence of inventory considerations. Chan et al. (2004) provide a comprehensive review of coordinated pricing and inventory models including markdown and clearance pricing. Our review is more aligned with Yano \& Gilbert (2003) in which inventory replenishment is critical. However, we do not survey EOQ-type models and deterministic models emphasizing demand smoothing (as a result of convex production cost) which are comprehensively surveyed in Yano \& Gilbert (2003). Neither are models involving initial inventory decisions in conjunction with markdown pricing decisions reviewed in Elmaghraby \& Keskinocak (2003) and Chan et al. (2004). On the other hand and more importantly, we cover many recent papers which appear after the publication of the three surveys. Our intention is not to provide a comprehensive review but rather to highlight key up-to-date developments and their historical roots.

The organization of this chapter is as follows. In the next section, we present commonly used demand models, which is followed by a survey of deterministic periodic review inventory and pricing models. We then present stochastic models distinguishing between single period models, multi-period models with convex ordering costs and multi-period models with concave ordering costs. Built upon the inventory and pricing models presented in the previous sections, we then review models on supply chain competition, coordination and cooperation. Finally, we provide some concluding remarks and thoughts on future research. 


\section{Demand Models}

To make optimal pricing decisions, it is pivotal to know the volume of a product that customers are willing to purchase at a specific price. The relationship between the volume and price gives rise to a demand model. Depending on the scenario, the demand of a product can depend on many variables other than price such as quality, brand name and competitor's prices. Here, however, we restrict our discussion to demand models in which price is the only variable.

Economic theory provides us with basic demand models derived from the classical rational theory of consumer choice (we refer to Van Ryzin, this volume and Chapter 7 in Talluri \& Van Ryzin, 2004 as well as the reference therein for more details). From this theory, it is typical to assume that the demand of a product is a decreasing function of its current price and revenue is concave as a function of price. Some commonly used deterministic demand functions include the linear demand $d(p)=b-a p$ for $p \in[0, b / a](a>0$ and $b \geq 0)$, the exponential demand $d(p)=e^{b-a p}(a>0$ and $b>0)$, the iso-price-elastic demand ${ }^{1} d(p)=a p^{-b}(a>0$ and $b>1)$, and the Logit demand $d(p)=N \frac{e^{-a p}}{1+e^{-a p}}$ which is the product of the market size $N$ and the probability that a customer with a coefficient of price sensitivity $a$ buys at price $p$.

We now present demand models for multiple products whose demands depend on prices of all products. Let $\boldsymbol{p}=\left(p_{1}, p_{2}, \ldots, p_{n}\right)$ be the price vector of $n$ products and $\boldsymbol{d}(\boldsymbol{p})=\left(d_{1}(\boldsymbol{p}), d_{2}(\boldsymbol{p}), \ldots, d_{n}\left(\boldsymbol{p}_{n}\right)\right)$ be the associated demand vector. As is consistent with demand models for a single product, we often assume that $\frac{\partial d_{i}(\boldsymbol{p})}{\partial p_{i}} \leq 0$, which implies that the demand of product $i$ is non-increasing in its own price. The sign of $\frac{\partial d_{i}(\boldsymbol{p})}{\partial p_{j}}(i \neq j)$ depends on whether products $i$ and $j$ are complements or substitutes. In the former case, $\frac{\partial d_{i}(\boldsymbol{p})}{\partial p_{j}} \leq 0$ while in the latter case, $\frac{\partial d_{i}(\boldsymbol{p})}{\partial p_{j}} \geq 0$.

Some specific multi-product demand models can be derived by extending demand functions of a single product. For instance, the linear demand model for multiple products, is given by $\boldsymbol{d}(\boldsymbol{p})=\boldsymbol{b}+\boldsymbol{A} \boldsymbol{p}$, where $\boldsymbol{b}=\left(b_{1}, b_{2}, \ldots, b_{n}\right)$ is the vector of coefficients and $\boldsymbol{A}=\left[a_{i j}\right]$ is an $n \times n$ matrix of price sensitivity coefficients with $a_{i i}<0$ and $a_{i j} \leq 0$ or $a_{i j} \geq 0(i \neq j)$ for complements or substitutes respectively. The exponential demand model is a composite of the exponential function and linear functions, given by $d_{i}(\boldsymbol{p})=e^{b_{i}+\boldsymbol{A}_{i}^{T} \boldsymbol{p}}, i=1,2, \ldots, n$. The iso-price-elastic demand model

\footnotetext{
${ }^{1}$ The price elasticity of demand is the relative change in demand in response to a relative change in price. It is defined for a demand function $d(p)$ as $e(p)=-\frac{p d^{\prime}(p)}{d(p)}$.
} 
can be represented as $d_{i}(\boldsymbol{p})=b_{i} p_{1}^{-a_{i 1}} p_{2}^{-a_{i 2}} \ldots p_{n}^{-a_{i n}}, i=1,2, \ldots, n$, where $a_{i j}$ defines the cross price elasticity between products $i$ and $j$. Finally, the Logit demand is given by $d_{i}(\boldsymbol{p})=N \frac{e^{-a_{i} p_{i}}}{1+\sum_{j=1}^{n} e^{-a_{j} p_{j}}}$ which is the product of the market size $N$ and the probability that a customer chooses product $j$ as a function of the price vector $\boldsymbol{p}$ (the probability is derived from the commonly used Multinomial Logit model).

In stochastic settings, the demand of a product is often represented as a function of the price $p$ and a random noise $\epsilon$ independent of $p$ denoted by $d(p, \epsilon)$. Sometimes it is important to specify the format that the random noise $\epsilon$ enters the demand function. If the demand is a deterministic function of price $p, d(p)$, plus the random noise $\epsilon$ (usually normalized to have zero mean), i.e., $d(p, \epsilon)=d(p)+\epsilon$, it is called an additive demand. If the demand is a deterministic function of price $p, d(p)$, multiplied by the random noise $\epsilon$ ( assumed to be nonnegative and usually normalized to have unit mean), i.e., $d(p, \epsilon)=d(p) \epsilon$, it is referred to as a multiplicative demand. More general demand functions of the following forms $d(p, \epsilon)=d_{1}(p) \epsilon+d_{2}(p)$ or $d(p, \epsilon)=d_{1}(p) \epsilon_{1}+\epsilon_{2}$ (where $\epsilon_{1} \geq 0$ and $\epsilon_{2}$ are two random variables) are also used in the inventory and pricing literature. Stochastic multi-product demand models can be also defined correspondingly.

Observe that for additive demand, the demand variance is independent of price while the coefficient of variation (the ratio of standard deviation and mean) is dependent of price. In contrast, for multiplicative demand, the coefficient of variation does not depend on price while the variance does. In single product settings with decreasing expected demand $d(p)$, higher price leads to higher uncertainty for additive demand but lower uncertainty for multiplicative demand.

The above demand models derived from economic theory assume that the demand of a product only depends on its current price. This assumption, appropriate to model impulsive purchasing, is unreasonable when consumers actively react to firms' dynamic pricing strategies, as demonstrated by plenty of empirical evidence.Modeling consumer behavior to explain and predict how individuals react to dynamic pricing strategies has been a very important research topic in the marketing literature (see Monroe, 2003). It has also received considerable attention in the operations management community in recent years (see Aviv and Vulcano, this volume for a review). Instead of providing a detailed review of the literature on consumer behavior models, we present in the next section one class of well-studied consumer behavioral pricing models in the marketing literature, the so-called reference price model, which has recently been incorporated into integrated inventory and pricing 
models.

\section{Periodic Review Deterministic Models}

In this section, we survey periodic review inventory and pricing models within deterministic settings. We first present a general modeling framework for a single product. Specifically, consider a firm that makes replenishment and pricing decisions of a single product over a finite planning horizon with $T$ periods. At the beginning of period $t(t=1,2, \ldots, T)$, the firm decides on its order quantity $x_{t}$, which incurs a fixed ordering cost $k_{t}$ (charged only if $x_{t}>0$ and independent of the order quantity) and a variable ordering cost $c_{t}$ per unit. In addition, an upper bound $q_{t}$ may also be imposed on the order quantity $x_{t}$. At the same time, the firm also determines its selling price $p_{t}$. Demand of period $t$ is assumed to be a deterministic function of the current period selling price $p_{t}$, denoted as $d_{t}\left(p_{t}\right)$. For simplicity, we assume that no backorder is allowed ${ }^{2}$ and leftover inventory is carried over from period $t$ to the next period incurring an inventory holding cost $h_{t}$ per unit. The firm's objective is to find a sequence of order quantities $x_{t}$ and prices $p_{t}$ so as to maximize the total profit over the planning horizon.

Upon denoting a pricing plan $\boldsymbol{p}=\left(p_{1}, p_{2}, \ldots, p_{T}\right)$ and its related demand sequence $\boldsymbol{d}(\boldsymbol{p})=$ $\left(d_{1}\left(p_{1}\right), d_{2}\left(p_{2}\right), \ldots, d_{T}\left(p_{T}\right)\right)$, a mathematical model for the deterministic inventory and pricing problem is:

$$
\begin{array}{ll}
\operatorname{Max} & \sum_{t=1}^{T} p_{t} d_{t}\left(p_{t}\right)-C(\boldsymbol{d}(\boldsymbol{p})) \\
\text { Subject to } & p_{t} \in\left[\underline{p}_{t}, \bar{p}_{t}\right], t=1,2, \ldots, T,
\end{array}
$$

where a lower bound $\underline{p}_{t}$ and an upper bound $\bar{p}_{t}$ on the selling price $p_{t}$ are imposed to prevent low profit margin and unreasonable high price respectively. In the objective function of the above problem, the first term is the total revenue, and the second term $C(\boldsymbol{d}(\boldsymbol{p}))$ is the minimum ordering and inventory holding cost over the planning horizon for a given pricing plan $\boldsymbol{p}$, which can be obtained

\footnotetext{
${ }^{2}$ Models with backorders can be handled following similar idea.
} 
by solving the following classical capacitated economic lot sizing model: for $\boldsymbol{d}=\left(d_{1}, d_{2}, \ldots, d_{T}\right)$,

$$
\begin{array}{ll}
C(\boldsymbol{d})=\begin{array}{l}
\text { Min } \\
\text { Subject to }
\end{array} & \sum_{t=1}^{T} k_{t} z_{t}+c_{t-1} x_{t}+h_{t} I_{t} \\
& I_{0}=0 \\
& x_{t} \leq d_{t} z_{t}, t=1,2, \ldots, T \\
& z_{t} \in\{0,1\}, t=1,2, \ldots, T \\
& I_{t}, x_{t} \geq 0, t=1,2, \ldots, T
\end{array}
$$

where the binary variable $z_{t}$ indicates whether an order is placed or not at period $t$ and the variable $I_{t}$ is the inventory level at the end of period $t$. In addition, the first constraint is the inventory balance equation, the second one states that we start with zero inventory, the third one is the capacity constraint (in case of no capacity on order quantity one can simply replace $q_{t}$ with an upper bound on the total demand from periods $t$ to $T$ ), and finally $I_{t} \geq 0$ implies that no backorder is allowed.

The major focus of this literature is on designing efficient algorithms for finding optimal solutions to problem (1) and its extensions by exploiting their structures. To illustrate the basic ideas involved, we sketch algorithms to solve problem (1) under different assumptions.

We first focus on settings with no capacity constraint. The key is to observe that without capacity constraint, there is an optimal ordering plan for problem (2) which has the so-called zeroinventory-ordering (ZIO) property, i.e., $x_{t} I_{t-1}=0$ or equivalently no order is placed whenever the inventory level is positive. To see this, consider an ordering plan which specifies for a period, say $t$, with a positive initial inventory level $I_{t-1}$, an order $x_{t}$ to be placed. Let $i$ be the ordering period immediately before period $t$. We can either shift the entire order placed at period $t$ to period $i$ or shift an order quantity of $I_{t-1}$ units from period $i$ to period $t$. In the first option, no order is place at period $t$ and the resulting cost change is $\left(c_{i t}-c_{t}\right) x_{t}$, while in the second option, the initial inventory level at period $t$ is zero and the resulting cost change $-\left(c_{i t}-c_{t}\right) I_{t-1}$, where $c_{i t}=c_{i}+\sum_{l=i}^{t-1} h_{l}$ is the marginal cost of satisfying period $t$ 's demand by an order placed at period $i$. Clearly one of the options leads to an ordering plan with a cost no more than that of the original plan. The same process is repeated on the plan with the lowest cost until we end up with a plan with the ZIO property in at most $T-1$ steps.

It is clear that for an ordering plan with the ZIO property, it suffices to specify the ordering 
periods. Specifically, if periods $i$ and $j(i<j)$ are two consecutive ordering periods in such an ordering plan, the ZIO property implies that the demand at period $t(i \leq t<j)$ is filled by the order placed at period $i$ only and thus the marginal cost of satisfying period $t$ 's demand is given by $c_{i t}$. The associated optimal price for period $t$ with $i \leq t<j$ can then be derived by finding the highest profit of period $t$ :

$$
\begin{array}{lll}
v_{i t}= & \operatorname{Max} & p_{t} d_{t}\left(p_{t}\right)-c_{i t} p_{t} \\
& \text { Subject to } \quad p_{t} \in\left[\underline{p}_{t}, \bar{p}_{t}\right] .
\end{array}
$$

In the case with no fixed ordering costs, it is straightforward to identify all ordering periods $t_{l}$ recursively by letting $t_{1}=1$ and $t_{l+1}=\min \left\{T+1, \min \left\{t: t \in\left(t_{l}, T\right], c_{t} \leq c_{t_{l}}\right\}\right\}$ for $l \geq 1$. In the case with fixed ordering costs, it is not sufficient to compare the marginal costs. However, the ZIO property allows us to construct an equivalent longest path problem in an acyclic network $G=(V, E)$ with the node set $V=\{1,2, \ldots, T+1\}$ and the arc set $E=\{(i, j): 1 \leq i<j \leq T+1\}$. In the construction, an arc $(i, j)$ means that the demands from periods $i$ up to $j-1$ are served by the order placed at period $i$. The length assigned to arc $(i, j)$ is $\sum_{t=i}^{j-1} v_{i t}-k_{i}$, i.e., the total profit from periods $i$ to $j-1$ with the prices determined by solving problem (3) for $t=i, \ldots, j-1$. One can show that a longest path from node 1 to node $T+1$ in the network $G$ gives an optimal ordering plan with the ZIO property whose ordering periods exactly correspond to the nodes on the longest path, and the associated optimal prices are derived through problem (3). The algorithm involves solving $T(T+1) / 2$ subproblems with the same structure as $(3)$ and then finding a longest path in the acyclic network $G$.

One way to find a longest path in the acyclic network $G$ is a forward-type algorithm which actually derives longest paths from node 1 to all nodes. Here is the sketch of the idea. For a given node $t(t=1,2, \ldots, T)$, once we have the longest paths from node 1 to nodes $1,2, \ldots, t-1$, a longest path from node 1 to node $t$ is the one with the longest length among $t-1$ options: following the longest path from node 1 to node $\tau$ and then arc $(\tau, t), \tau=1,2, \ldots, t-1$. The computational complexity of the forward-type algorithm is $O\left(T^{2}\right)$, i.e., there exists a constant $\rho>0$ such that the algorithm solves the problem in no more than $\rho T^{2}$ elementary operations (additions, multiplications and comparisons $)^{3}$.

Wagner \& Whitin (1958a) appear to be the first to incorporate the pricing decision into the

\footnotetext{
${ }^{3}$ We refer to Ahuja et al. (1993) for more details on solving longest (shortest) path problems in acyclic networks.
} 
now classical economic lot sizing model without capacity constraint (also commonly referred to as the Wagner-Whitin model; see Wagner \& Whitin, 1958b). They recognize the ZIO property and sketch a forward-type algorithm similar to the one described here. They also suggest to use the planning horizon property (i.e., if for a problem with $t$ periods an order is placed at period $t$, then all periods prior to $t$ can be ignored in determining the optimal policy for future periods) to simplify the computation. These results are parallel to the ones in the Wagner-Whitin model (see Wagner \& Whitin, 1958b) ${ }^{4}$. Thomas (1970) analyzes a model similar to Wagner \& Whitin (1958a) and illustrates explicitly that an optimal price at period $t$ can be identified, independent of other periods' prices, by solving the single variable optimization problem (3) if demand of period $t$ is satisfied by an order placed at period $i$. Geunes et al. (2006) consider several extensions by allowing for multiple price-demand curves and piecewise linear concave ordering costs and illustrate these extensions can be handled easily by adapting the algorithm sketched here without substantially increasing the computational complexity. They also present an equivalent integer program formulation for problem (1) whose linear programming relaxation does not have an integrality gap ${ }^{5}$ under the assumption that the revenue at each period is a piecewise linear (with finite pieces) and concave function in terms of the satisfied demand.

We now focus on problem (1) with capacity constraints. We start with the case with no fixed ordering costs. Assume that the demand function $d_{t}\left(p_{t}\right)$ is strictly decreasing in the selling price $p_{t}$, which implies that there is a one-to-one correspondence between the demand and the selling price at period $t$. Thus, solving problem (1) is equivalent to determining a sequence of demands that gives the maximum profit, and in this case, problem (1) can be formulated as a min-cost network flow problem ${ }^{6}$. If, in addition, the revenue curves are assumed to be concave, it can be

\footnotetext{
${ }^{4}$ Interestingly, Wagner \& Whitin (1958a) focusing on the economic lot sizing model with price-dependent demand precede Wagner \& Whitin (1958b) which analyzes the economic lot sizing model without pricing decisions; see Wagner (2004) for a recollection of the background. In addition, surprisingly, Wagner \& Whitin (1958a) do not refer to Whitin (1955), which, analyzing the newsvendor model with price-dependent demand, appears to be the first attempt incorporating pricing decisions into inventory models.

${ }^{5}$ Similar integer program formulations for economic lot sizing models without pricing decisions are powerful in designing efficient algorithms for models with multiple products (see Pochet \& Wolsey, 2006).

${ }^{6}$ We first construct a directed network $G=(V, E)$, in which the node set $V=\{0,1, \ldots, T, T+1\}$ and the arc set $E=\{(t, t+1): t=1,2, \ldots, T-1\} \cup\{(0, t),(t, T+1): t=1,2, \ldots, T\} \cup\{(T+1,0)\}$. The flow on arc $(0, t)(t=$ $1,2, \ldots, T)$, denoted by $x_{t}$, belongs to $\left[0, q_{t}\right]$ and incurs a linear cost $c_{t} x_{t}$. The flow on $\operatorname{arc}(t, t+1)(t=1,2, \ldots, T-1)$, denoted by $I_{t}$, is nonnegative and incurs a linear cost $h_{t} I_{t}$. The flow on arc $(t, T+1)(t=1,2, \ldots, T)$, denoted by
} 
solved efficiently by standard algorithms for min-cost network flow problems with convex cost (see Ahuja et al., 1993). It can also be solved by an intuitive and interesting greedy algorithm. Assuming integer-valued inventory levels, order quantities and demand, the greedy algorithm works as follows: starting with zero demand, at each iteration, identify a period and increase its demand by one unit if it respects capacity constraints and gives the highest profit increase among all possible periods; stop the process if no further improvement is possible. The greedy algorithm is proposed by Biller et al. (2005), who also present computational experiments suggesting that it is possible to achieve significant benefit with few price changes.

The capacitated inventory and pricing model with fixed ordering costs is not significantly different from the capacitated economic lot sizing (CELS) model (2) (see Geunes et al., 2006 for the model with piecewise linear concave revenue functions, Deng \& Yano, 2006 and Geunes et al., 2009 for the model with general concave revenue functions - all are with respect to the satisfied demand). Optimal ordering plans may not have the ZIO property anymore for the capacitated models. Instead, it can be shown that there exists an optimal ordering plan consisting of a series of capacity constrained production sequences. That is, we can partition the planning horizon so that each member of the partition (called a production sequence) consists of a series of consecutive periods that starts and ends with zero inventory level, and holds positive inventory in between, and at each period of the production sequence, we either produce nothing or to the full capacity, except for at most one period (referred to as the fractional production period) at which one can produce a quantity strictly between zero and the full capacity. To prove this statement, observe that model (2) is a concave minimization problem with linear constraints and thus attains optimality at some extreme point, which exactly corresponds to an ordering plan consisting of a series of capacity constrained production sequences.

We can determine the optimal prices associated with any given production sequence independent of other production sequences. To simplify our presentation, assume that all inventory holding costs are zero (note that this can be assumed without loss of generality since we can reformulate problem (1) by replacing inventory variables $I_{t}$ by $\sum_{l=1}^{t}\left(x_{l}-d_{l}\right)$ and rearranging terms). Under $d_{t}$, belongs to the interval $\left[d_{t}\left(\bar{p}_{t}\right), d_{t}\left(\underline{p}_{t}\right)\right]$ and incurs a cost $-d_{t} g_{t}\left(d_{t}\right)$, where $g_{t}(\cdot)$ is the inverse function of $d_{t}(\cdot)$. The flow on arc $(T+1,0)$ is nonnegative and does not incur any cost. With this construction, problem (1) is equivalent to finding a circulation in the network $G$ with a minimum cost. 
this assumption, the key observation is that at optimality the marginal revenue (with respect to the satisfied demand) at each period within a production sequence is essentially the same (we say "essentially" because exceptions happen when we take care of the boundary conditions on prices) and we can look at three different cases: (a) if the fractional production period is specified, the optimal price at each period can be easily determined by setting its marginal revenue equal to the variable production cost of the fractional production period; (b) if there is no fractional production period but there is a period, called a fractional demand period, in which the price does not attain the bound constraints, the marginal revenue at each period equals the marginal revenue at the fractional demand period, and if in addition we know the total production quantity of the production sequence, we can then determine the optimal prices; (c) if there is no fractional production period nor fractional demand period, we can determine whether the optimal prices are at their lower bounds or upper bounds by comparing the marginal revenues at these bounds. If we have equal capacities $q_{t}$ at all periods, we can find the optimal production plans like what we do for the CELS problem for all cases (a)-(c) and thus solve problem problem (1) in polynomial time. Of course, in case (b), we first need to determine optimal price vector candidates. However, the number of price vector candidates is equal to the number of possible total production quantities which is no more than the number of periods of the production sequence. If the capacities $q_{t}$ are time dependent, even the CELS problem is NP-hard (see Florian \& Morton, 1971; Florian et al., 1980 for the CELS model with equal and unequal capacities respectively).

The models analyzed above assume that prices can be freely changed from a period to the next. However for certain products like catalog goods, firms may want to determine a price at the beginning of the selling season and maintain the same price throughout the whole planning horizon. We can use problem (1)-(2) to model such a setting by simply adding constraints $p_{1}=\ldots=p_{T}$ and end up with the so-called joint inventory and static pricing model. A heuristics for solving the model is the alternating coordinates minimization (ACM) type algorithm. That is, for a given price (and hence a given demand process) find an optimal ordering plan by solving an economic lot sizing problem; then for this ordering plan, find an associated optimal price using the approach we sketched earlier. The ACM algorithm alternates between these two steps until no further improvement is possible.

Kunreuther \& Schrage (1973) were the first to propose and analyze the joint inventory and 
static pricing problem with no capacity constraint. They assume that the demand at period $t$ takes the form $d_{t}(p)=b_{t}+a_{t} d(p)$ for some nonnegative coefficients $a_{t}, b_{t}$, in which the function $d(p)$ is a non-increasing differentiable function. They propose to apply the ACM algorithm starting from a lower bound $\underline{p}$ and a upper bound $\bar{p}$ of price separately. Let $p_{l}^{*}$ and $p_{u}^{*}$ be the corresponding terminating prices after a finite number of steps. They prove that their approach does not skip any optimal solution in $\left[\underline{p}, p_{l}^{*}\right]$ and $\left[p_{u}^{*}, \bar{p}\right]$ (i.e., the profit of a newly generated price is always no less than the profit of any price between this newly generated price and the price generated in the previous step) and thus an optimal price lies in the interval $\left[p_{l}^{*}, p_{u}^{*}\right]$. van den Heuvel \& Wagelmans (2006) propose to restart the approach in Kunreuther \& Schrage (1973) whenever there are unexplored intervals. They argue that the minimum total ordering and inventory holding cost is a piecewise linear concave function of the price effect $d(p)$ with at most $O\left(T^{2}\right)$ linear pieces and their algorithm involves solving $O\left(T^{2}\right)$ economic lot sizing problems each of which corresponds to a linear piece, which implies that the joint inventory and static pricing problem with no capacity constraint can be solved in polynomial time. The capacitated counterpart of Kunreuther and Shrage's model is analyzed in Geunes et al. (2009) (in addition to the capacitated inventory and pricing model with dynamic pricing).

Gilbert (1999) considers a special case of the model proposed by Kunreuther \& Schrage (1973) with the assumption that the cost parameters $c_{t}, h_{t}$ and $k_{t}$ are time independent and the demand takes the form $d_{t}(p)=a_{t} d(p)$. A key observation from these assumptions is that given the number of set-ups, say $n$, the minimum ordering and inventory holding cost (even though the number of set-ups is given, we still need to identify the ordering periods) is a linear function of the price effect $d(p), n K+\gamma_{n} d(p)$, where the coefficient $\gamma_{n}$ is independent of $p$ and can be derived by solving a simple dynamic program in $O\left(T^{2}\right)$ time. Since there are $T$ possible candidates for the number of set-ups, Gilbert (1999) shows that his model can be solved in $O\left(T^{3}\right)$ time under the additional assumption that the function $d(\cdot)$ has an inverse $p(d)$ and the revenue function $d p(d)$ is concave.

A predominant assumption made in the existing inventory and pricing literature is that price adjustment is costless. Yet, various empirical studies illustrate that price adjustment costs do exist and play a crucial role in shaping firms' pricing strategies. For instance, Levy et al. (1997) report that price adjustment may generate enormous costs for major retailer chains, and take up as much as $40 \%$ of the reported profits for some of these chains. A few papers that take into account price 
adjustment cost include Chen \& Hu (2008) and Chen et al. (2008), which analyze a stochastic model and will be reviewed in the next section.

Chen \& Hu (2008) assume that a cost of changing price tags, independent of the magnitude of the price change, is charged if price at a period is different from its previous period. As both fixed ordering cost and price adjustment cost are taken into account, their model is much more complicated. Still, under the assumption of no capacity constraint, they develop exact algorithms for finding the optimal order quantities and selling prices. The idea is to partition the planning horizon such that each member of the partition consists of consecutive periods with a constant price. The total profit is then appropriately allocated to each member of the partition, and for each member they solve a joint inventory and static pricing problem to find a single constant price maximizing its allocated profit. Based on this result, Chen \& Hu (2008) construct an acyclic network so that solving their inventory and pricing model with price adjustment cost is equivalent to finding a longest path in the acyclic network.

A few papers start to relax the assumption that demand at a period depends only on the current period's price. In the deterministic setting, Ahn et al. (2007) study an inventory and pricing model in which the ordering cost is linear and the demand at a period depends on the prices of the current period and previous periods. Specifically, they assume that at each period, new customers enter the market and a fraction of them may stay for certain fixed periods. The amount of new customers is a linear function of the current period's price. This model allows them to capture the size of customer segment for a given valuation. If the price drops down to a level below the valuation of a customer in the market, the customer makes a purchase and leaves the market. The total demand at a period is then represented as a function of the prices of its current period as well as its previous periods. The authors develop effective heuristics for their optimization problem. When there is no capacity constraint and customers stay for at most one more period in addition to the period when they enter the market, they derive closed-form solutions for the case with stationary parameters and develop an efficient polynomial time algorithm for the case with non-stationary parameters.

Chen et al. (2009a) propose and analyze a deterministic finite horizon coordinated pricing and inventory model in which demand functions are specified by a class of reference price models. In such models, it argues that consumers develop price expectations, referred to as reference prices, 
and use them to judge the purchase price of a product (see Mazumdar et al., 2005 for a review). Among many different reference price models, a memory-based model, in which reference price is the weighted average of a product's own past prices, is commonly used and empirically validated on scanner panel data for a variety of products (see Greenleaf, 1995). Specifically, in an exponentialsmoothing memory-based model, the reference price at period $t, r_{t}$ is determined as: $r_{t}=\alpha r_{t-1}+$ $(1-\alpha) p_{t-1}$, where $p_{t-1}$ is the previous period price and $\alpha \in[0,1]$ is the smoothing factor.

The demand at period $t$, taking into account the effect of reference price, can be modeled as a kinked demand curve, namely $d_{t}\left(p_{t}, r_{t}\right)=b_{t}-a_{t} p_{t}+f\left(r_{t}-p_{t}\right)$ (see Fibich et al., 2003; Greenleaf, 1995; Kopalle et al., 1996). Here $a_{t}$ and $b_{t}$ are some given positive constants while $f(u)=\delta u$ for $u>0$ and $f(u)=\gamma u$ for $u<0$ with $0 \leq \delta \leq \gamma$. The definition of $f$ implies that the effect of $r_{t}-p_{t}$ on demand is asymmetric and consumers are loss averse, i.e., consumers are more sensitive to loss $\left(p_{t}>r_{t}\right)$ than gain $\left(p_{t}<r_{t}\right)$. Such a demand function is not only empirically validated but also supported by Kahneman \& Tversky (1979)'s prospect theory (see Mazumdar et al., 2005 for more discussion and Popescu \& Wu, 2007 and Nasiry \& Popescu, 2008 for extensions of the reference models).

The revenue function $p_{t} d_{t}\left(p_{t}, r_{t}\right)$ may not be jointly concave in $p_{t}$ and $r_{t}$. Nevertheless, Chen et al. (2009a) develop strongly polynomial time algorithms for the cases with no fixed ordering costs under some technical conditions and propose a heuristic for the general case with an error bound estimation. Their numerical study illustrates that incorporating reference price effect into integrated inventory and pricing models can have a significant impact on firms' profits. In addition, the more reference price effect contributing to the demand, the larger the benefit of pricing and inventory integration.

All the above papers focus on a single product. Gilbert (2000) considers a multi-product inventory and pricing model in which there is a joint production capacity for all products and fixed ordering cost is negligible. Assuming that the revenue of a product is concave in demand intensities, the author develops an algorithm to find the optimal solution by exploiting the special problem structure, which unfortunately may not run in polynomial time. Hall et al. (2003) develop an inventory and pricing model for products of a category with joint fixed ordering costs in which demand of a product depends on the prices of all products in the category. They develop a dynamic programming formulation to solve their model and demonstrate the benefits obtained via a category 
management approach.

\section{Stochastic Models}

In this section, we focus on integrated inventory and pricing models with stochastic demand. We will first review single period models, which are appropriate for products with long production/order lead times but short selling seasons, and then move to multi-period models in which replenishment is possible in the planning horizon.

\section{Single Period Models}

A single period inventory and pricing model with stochastic demand is an extension of the classical newsvendor model and is thus often referred to as a newsvendor model with price dependent demand. In this model, at the beginning of the period, an order with a quantity of $x$ units is placed incurring a per-unit cost $c$. At the same time, a selling price $p$ is decided. During the period, a stochastic price dependent demand, denoted as $d(p, \epsilon)$ with $\epsilon$ being a random perturbation, is realized. If the order quantity $x$ is larger than the realized demand $d(p, \epsilon)$, a per-unit holding cost $h$ is charged for excess inventory (if $h<0$, then it corresponds to the salvage value). On the other hand, if the realized demand $d(p, \epsilon)$ is larger than the order quantity, excess demand is lost incurring a unit penalty cost $b$. The objective is to decide on the order quantity $x$ and the selling price $p$ so that the expected profit is maximized. Upon denoting $x^{+}=\max (x, 0)$, the newsvendor model with price dependent demand is formulated as follows:

$$
\operatorname{Max}_{x \geq 0, p \in[\underline{p}, \bar{p}]} p \mathrm{E}[\min (d(p, \epsilon), x)]-c x-\mathrm{E}\left[h(x-d(p, \epsilon))^{+}+b(d(p, \epsilon)-x)^{+}\right],
$$

where the first term is the expected revenue, the second term is the ordering cost, and the last term is the expected inventory holding cost and lost sales cost.

To simplify our presentation, we use $d(p, \epsilon)=d_{1}(p) \epsilon+d_{2}(p)$ (referred to as the mixture demand model), where $d_{1}(p)$ and $d_{2}(p)$ are two deterministic functions of price and $\epsilon$ is a nonnegative random variable. Note that when $d_{1}(p)=1$, the mixture demand model is the additive demand model, and when $d_{2}(p)=0$, it is the multiplicative demand model. Let $d(p)=d_{1}(p) \mathrm{E}[\epsilon]+d_{2}(p)$ be the expected demand for a given $p$ and $R(p)=(p-c) d(p)$, the profit function of the deterministic 
counterpart of the newsvendor model with price dependent demand. Define $z=\frac{x-d_{2}(p)}{d_{1}(p)}$, which can be interpreted as the stocking factor representing a surrogate for safety stock factor, the number of standard deviation that stocking quantity deviates from expected demand (see Petruzzi \& Dada, 1999). Problem (4) is equivalently reformulated as

$$
\operatorname{Max}_{z, p} \phi(z, p)=R(p)-(p-c) d_{1}(p) u(z)-d_{1}(p) v(z),
$$

where $u(z)=\mathrm{E}[\epsilon]-\mathrm{E}[\min (z, \epsilon)]$ and $v(z)=(c+h) z+b \mathrm{E}[\epsilon]-(c+b+h) \mathrm{E}[\min (z, \epsilon)]$. It is clear that $u(z) \geq 0$ and $v(z) \geq 0$ for all $z$. Assume that the cumulative distribution function of $\epsilon, F(\cdot)$, is continuous. Under this assumption, there is a unique solution $z$, denoted by $z^{*}(p)$, maximizing $\phi(z, p)$ for a given $p$. In fact, $z^{*}(p)=F^{-1}\left(\frac{p+b-c}{p+b+h}\right)$ is the optimal solution for a corresponding newsvendor model. Let $\pi(p)=\phi\left(z^{*}(p), p\right)$ be the induced expected profit function. The optimal price can then be solved by maximizing $\pi(p)$. Unfortunately, the profit function $\pi(p)$ may not be concave or quasi-concave.

Thus, one of the major focuses in the literature concerns structural results of the optimization problem (4), namely the existence and uniqueness of the optimal solutions and concavity or quasiconcavity of the expected profit functions, and comparative statics analysis. We first consider the deterministic counterpart. Note that a stationary point of $R(p)$ satisfies

$$
p\left(1-\frac{1}{e(p)}\right)=c .
$$

Recall that $e(p)=-p d^{\prime}(p) / d(p)$ is the price elasticity of the expected demand. If the expected demand $d(p)$ has an increasing price elasticity, which includes linear demand and concave demand, then $R(p)$ has a unique stationary point and thus is quasi-concave in $[c, \bar{p}]$. The newsvendor model with price dependent demand can also be handled by analyzing the stationary point of the induced expected profit function $\pi(p)$. Since the derivation is rather tedious, we simply present the results (more details can be found in Yao et al., 2006). Essentially, if the expected demand has an increasing price elasticity and the random variable $\epsilon$ has an increasing generalized failure rate for the multiplicative demand case or an increasing failure rate for the additive demand case $^{7}$, then $\pi(p)$ has a unique stationary point and therefore is quasi-concave in $[c, \bar{p}]$.

\footnotetext{
${ }^{7}$ Let $f(\cdot)$ be the density of the cumulative demand distribution $F(\cdot)$. The distribution has an increasing (generalized) failure rate if its failure rate function $\frac{f(\xi)}{1-F(\xi)}$ (or correspondingly its generalized failure rate function $\frac{\xi f(\xi)}{1-F(\xi)}$ ) is increasing. This includes several commonly used distributions such as uniform, (truncated) Normal, exponential, Gamma distributions.
} 
Another major focus of the literature concerns the relation between stochastic model and its deterministic counterpart. Interestingly, the relation of the optimal price in a newsvendor model with price dependent demand (referred to optimal risky price) and the optimal price of its deterministic counterpart (a price maximizing $R(p)$ and referred to as the optimal riskless price) depends on how uncertainty is incorporated into the demand function. Specifically, if the demand is additive, i.e., $d(p, \epsilon)=d(p)+\epsilon$, then the optimal risky price is no more than the optimal riskless price. On the other hand, if the demand is multiplicative, i.e., $d(p, \epsilon)=d(p) \epsilon$, then the opposite is true, namely the optimal risky price is no less than the optimal riskless price.

We now sketch the analysis. Assume that $R(p)$ and $\pi(p)$ are quasi-concave and have unique stationary points in $[c, \bar{p}]$. The optimal riskless price $p^{0}$ and the optimal risky price $p^{*}$ are given by $R^{\prime}\left(p^{0}\right)=0$ and $\pi^{\prime}\left(p^{*}\right)=0$ respectively. From the envelope theorem,

$$
\pi^{\prime}(p)=\left.\frac{\partial \phi(z, p)}{\partial p}\right|_{z=z^{*}(p)}
$$

Let $z^{*}=z^{*}\left(p^{*}\right)$. We have that

$$
0=\pi^{\prime}\left(p^{*}\right)=R^{\prime}\left(p^{*}\right)\left(1-u\left(z^{*}\right) w\left(p^{*}\right)\right)-R\left(p^{*}\right) u\left(z^{*}\right) w^{\prime}\left(p^{*}\right)-d_{1}^{\prime}\left(p^{*}\right) v\left(z^{*}\right),
$$

where $w(p)=\frac{d_{1}(p)}{d_{1}(p) \mathrm{E}[\epsilon]+d_{2}(p)}$. Therefore,

$$
R^{\prime}\left(p^{*}\right)\left(1-u\left(z^{*}\right) w\left(p^{*}\right)\right)=R\left(p^{*}\right) u\left(z^{*}\right) w^{\prime}\left(p^{*}\right)+d_{1}^{\prime}\left(p^{*}\right) v\left(z^{*}\right) .
$$

Note that $1-u\left(z^{*}\right) w\left(p^{*}\right)>0$.

If both $d_{1}(p)$ and $w(p)$ are decreasing, then $R^{\prime}\left(p^{*}\right) \leq 0$ and the quasi-concavity of $R(p)$ implies that $p^{*} \geq p^{0}$. On the other hand, if both $d_{1}(p)$ and $w(p)$ are increasing, then $R^{\prime}\left(p^{*}\right) \geq 0$ and the quasi-concavity of $R(p)$ implies that $p^{*} \leq p^{0}$. As a consequence, we have $p^{*} \geq p^{0}$ for the additive demand model in which $d_{1}(p)=1$ and $d_{2}(p)$ is decreasing and $p^{*} \leq p^{0}$ for the multiplicative demand model in which $d_{2}(p)=0$ and $d_{1}(p)$ is decreasing.

To provide some intuition to the interesting comparison between the additive demand and the multiplicative demand, observe that price provides an opportunity to reduce the risk of overstocking and understocking as a result of demand uncertainty. Thus, it seems reasonable to choose a price to reduce both measures of demand uncertainty if possible: the demand variance $d_{1}^{2}(p) \sigma^{2}$ and coefficient of variation $w(p) \sigma=\frac{d_{1}(p) \sigma}{d_{1}(p) \mathrm{E}[\epsilon]+d_{2}(p)}$, where $\sigma$ is the standard deviation of $\epsilon$. If both 
measures are decreasing in price, one can expect the optimal risky price to be no less than the optimal riskless price. On the other hand, if both measures are increasing in price, the opposite is true.

The newsvendor model with price dependent demand was first proposed by Whitin (1955). He also sketches a sequential procedure to calculate the optimal price (first compute the optimal ordering quantity for a give price and then find the optimal solution of the induced expected profit function).

Papers that touch upon conditions for the existence and uniqueness of the optimal solution and concavity properties of the expected profit include Zabel (1970) (multiplicative demand), Young (1978) (mixture demand), Polatoglu (1991) (general demand), Petruzzi \& Dada (1999) (both additive and multiplicative demand), Yao et al. (2006) (both additive and multiplicative demand), Chen et al. (2006) (additive demand), Song et al. (2009) (multiplicative demand) and Kocabiykoglu \& Popescu (2009) (general demand). Among them, Yao et al. (2006) provide a nice summary of different conditions in many papers and also derive the conditions presented here. Chen et al. (2006) provide conditions for the quasi-concavity of the single period induced expected profit as a function of the order-up-to level for the additive demand case while Song et al. (2009) derive conditions for the concavity of the single period induced expected profit as a function of the order-up-to level for the multiplicative demand case. These conditions, to be discussed in details in the next subsection on multi-period stochastic models, involve assumptions on the expected demand and the failure rate function of the underlying random variable. Roughly speaking, they impose conditions that are slightly stronger than the concavity of the expected demand and assume that the underlying random variable satisfies properties closely related to the increasing failure rate requirement.

Recognizing that existing results and techniques depend heavily on how uncertainty is incorporated in the demand model, Kocabiykoglu \& Popescu (2009) attempt to propose a unified framework for general demand by introducing a new concept called the elasticity of lost sales. Let $q(p, x)$ be the probability that the demand $d(p, \epsilon)$ exceeds the inventory level $x$ for a given $p$ and $x$. The elasticity of lost sales is defined for a selling $p$ and inventory level $x$ as $-p \frac{\partial q(p, x)}{\partial p} / q(p, x)$. Kocabiykoglu \& Popescu (2009) illustrate that the concavity and submodularity of the expected profit and monotonicity of the optimal solutions are characterized by the monotonicity or bounds on the elasticity of lost sales, which are satisfied by most demand models analyzed in the literature. 
A different demand model is proposed in Raz \& Porteus (2006), in which the stochastic demand is specified by several fractiles. These fractiles are represented as piecewise linear functions of the selling price (with the same breakpoints for all fractiles). They argue that such demand models are tractable and allow them to capture certain settings in which additive or multiplicative demand is not appropriate, for instance, settings in which the lowest demand variability comes at either very high or very low prices or settings in which one has a good understanding of the market demand for a middle range of prices. They also illustrate that the optimal price may not be a monotone function of the ordering cost in contrast to most single period inventory and pricing models.

The relation between the optimal riskless price and the optimal risky price was first illustrated in Mills (1959) for the additive demand case. The multiplicative demand case was proven by Karlin \& Carr (1962). They also extend their single period model to an infinite horizon setting, in which a single constant price is specified at the beginning of the planning horizon, and prove that the optimal price lies between the optimal riskless price and the optimal risky price (both for a single period) if demand is multiplicative with a uniformly distributed perturbation or demand is additive.

Hempenius (1970) studies a problem similar to the one in Mills (1959) with an additive demand and obtains the same result regarding the optimal risky and riskless prices. The author also investigates how ordering and pricing decisions depend on the variance of the additive random perturbation. In addition, models taking into account risk aversion (using a mean-variance utility) and additive price-dependent random perturbation are proposed (most results are derived for specific demand distributions and sometimes numerically).

The comparison of the optimal riskless and risky prices for the mixture demand model presented here is carried out in Young (1978). Young (1979) extends this demand model by introducing a new parameter $b$ in the function $d_{1}(p)$, which the author argues represents competitiveness. The author performs comparative statics analysis of the optimal price, expected sales and average inventory and shortage costs with respect to the parameter $b$ and illustrates uncertainty may lead to results different from its deterministic counterpart.

The intuition explaining the observation of the optimal riskless and risky prices under different demand models is offered in Zabel (1972) and Petruzzi \& Dada (1999). The latter paper introduce a new price benchmark, referred to as the base price. For a given value of $z$, the base price is defined 
as the price maximizing the profit from expected sales, i.e., the optimal solution of the problem

$$
\operatorname{Max}_{p}(p-c)\left(d_{1}(p) \mathrm{E}[\min (z, \epsilon)]+d_{2}(p)\right) .
$$

Petruzzi \& Dada (1999) illustrate that the optimal risky price can be interpreted as the sum of the base price and a (nonnegative) premium for both the additive and multiplicative cases. Interestingly, in the additive demand case, the premium is zero since for a given $z$ the expected understocking and overstocking are independent of the price $p$.

All the above papers assume that unsatisfied demand is lost. Simchi-Levi et al. (2005) analyze a single period inventory and pricing model in which unsatisfied demand is filled through an emergency order with a cost higher than the regular ordering cost $c$. In this case, the expected profit function is well-behaved and existence and uniqueness can be warranted under general assumptions. Simchi-Levi et al. (2005) prove that for the multiplicative demand case, the optimal risky price is no less than the optimal riskless price, which is consistent with the lost sales models. However, for the additive demand case, the optimal risky price equals the optimal riskless price.

Most papers assume that demand depends only on price but not a firm's inventory level. Dana \& Petruzzi (2001) depart from this assumption by modeling customers' choice explicitly in view of product availability and their outside options, which leads to stochastic price and inventory dependent demand. They illustrate that by internalizing the inventory effect on demand, the firm would order more, set a higher fill rate and earn higher expected profit.

Several papers incorporate risk aversion into single period inventory and pricing models. Among them, Leland (1972) develops a model of an expected utility maximizing firm facing random demand whose relation with price is specified as an implicit function. Depending on whether the ordering and pricing decisions are made before or after the realization of the underlying uncertainty, Leland (1972) analyzes four models with different postponement strategies and compares risk averse models with their risk neutral counterparts.

Agrawal \& Seshadri (2000) analyze a model similar to the one in Simchi-Levi et al. (2005). However, they assume that the decision maker is risk averse and use expected utility as the risk measure. They prove that for the multiplicative demand case, the risk averse decision maker sets a higher price and orders less compared with its risk neutral counterpart, while for the additive demand case, the risk averse decision maker sets a lower price. A different risk averse model is 
analyzed in Chen et al. (2009b), in which risk is measured by the conditional value-at-risk, a risk measure commonly used in finance literature. Focusing on the lost sales model, they derive results regarding optimal risky price and riskless price that are consistent with risk neutral models. They also illustrate that the more risk averse the decision maker is, the smaller the optimal price will be (for both additive and multiplicative demand case), and the smaller the ordering quantity will be (for multiplicative demand case). A different and yet related model is proposed in Lau \& Lau (1988) in which the objective is to maximize the probability of attaining a target profile.

Research on multi-product models is limited. This is not very surprising given the analytical complexity even for single product models. Aydin \& Porteus (2008) further demonstrate the analytical complexity through a single period inventory and pricing problem with multiple products under price-based substitution and lost sales assumptions. They assume that a product's demand is a deterministic function of the prices with a multiplicative random perturbation, in which the deterministic functions are given by the attraction model from the marketing literature. Specifically, the demand for product $i$ is given by $d_{i}(\boldsymbol{p})=\frac{v_{i}\left(p_{i}\right)}{v_{0}+\sum_{j=1}^{n} v_{j}\left(p_{j}\right)} \epsilon_{i}$, where $n$ is the number of products, $v_{0}$ is a positive scalar and for $i=1,2, \cdots, N, v_{i}\left(p_{i}\right)$ are strictly decreasing in $p_{i}$ and $\epsilon_{i}$ are identically and independently distributed nonnegative random variables with an increasing failure rate cumulative distribution function. Aydin \& Porteus (2008) show that the objective function is not necessarily jointly quasi-concave in prices even for deterministic demand. Interestingly, under some technical conditions on the functions $v_{i}, i=1,2, \ldots, N$, they prove that their model admits a unique solution to the first order optimality condition and thus the solution is optimal.

Finally, we mention several papers that compare different postponement strategies in single period inventory and pricing models. van Mieghem \& Dada (1999) analyze two stage decision models (under monopoly, duopoly, or oligopoly with perfect competition) involving capacity, inventory and pricing decisions of a single product, Bish \& Wang (2004) and Chod \& Rudi (2005) study resource investment in settings with flexible resources and responsive price, and Tomlin \& Wang (2008) focus on a co-production system with two products. 


\section{Multi-period Models with Convex Ordering Costs}

In this and the next subsections, we review stochastic inventory and pricing models with multiple replenishment opportunities. The focus is on characterizing the structure of the optimal policies that provides important managerial insights and facilitates efficient computation. We first present a general model and then review relevant papers on models with convex ordering costs in this subsection and on models with concave ordering costs in the next subsection.

The sequence of events of the multi-period stochastic model is similar to its deterministic counterpart presented in the previous section. That is, at the beginning of period $t(t=1,2, \ldots, T)$, the firm decides on its order quantity and selling price for period $t$. Demand in period $t$ is assumed to be a stochastic function of the current period selling price $p_{t}$, denoted as $d_{t}\left(p_{t}, \epsilon_{t}\right)$, and is realized after the ordering and pricing decision for period $t$ is made. Let $x_{t}$ be the inventory level at the beginning of period $t$, just before placing an order. Similarly, $y_{t}$ is the inventory level at the beginning of period $t$ after placing an order. For tractability, order lead time is assumed to be zero.Thus, the ordering cost is a function of the order quantity $y_{t}-x_{t}$ and denoted as $C_{t}\left(y_{t}-x_{t}\right)$.

Since demand is stochastic, it is unlikely that all demands can be filled immediately from on hand inventory. In this case, unsatisfied demand is assumed to be either backlogged or lost; both the backlogging model and the lost sales model are commonly used in the literature. In the backlogging model, the selling price for the backlogged demand is the price in the period at which the demand occurs. Let $x$ be the inventory level carried over from period $t$ to period $t+1$. A cost $h_{t}(x)$ is incurred at the end of period $t$ which represents inventory holding cost when $x>0$ and backorder cost or lost sale penalty cost if $x<0$ depending on whether unsatisfied demand is backlogged or lost. $h_{t}(x)$ is usually assumed to be convex.

The objective is to decide on ordering and pricing policies to maximize total expected profit over the entire planning horizon. That is, to choose $y_{t}$ and $p_{t}$ to maximize

$$
\mathrm{E}\left[\sum_{t=1}^{T}-C_{t}\left(y_{t}-x_{t}\right)-h_{t}\left(y_{t}-d_{t}\left(p_{t}, \epsilon_{t}\right)\right)+p_{t} d_{t}\left(p_{t}, \epsilon_{t}\right)\right],
$$

where $x_{t+1}=\psi\left(y_{t}-d_{t}\left(p_{t}, \epsilon_{t}\right)\right)$. Here, $\psi(x)=x$ for the backlogging model and $\psi(x)=\max (x, 0)$ for the lost sales model.

We denote by $v_{t}(x)$ the maximum expected profit starting from the beginning of time period 
$t$ with inventory level $x$ to the end of the planning horizon $\left(v_{t}(x)\right.$ is referred to as the profit-to-go function). We write a recursion of $v_{t}(x)$ as a dynamic program as follows. For $t=T, T-1, \ldots, 1$, $v_{t}(x)=\operatorname{Max}_{y \geq x, \underline{p}_{t} \leq p \leq \bar{p}_{t}}-C_{t}(y-x)-\mathrm{E}\left[h_{t}\left(y-d_{t}\left(p, \epsilon_{t}\right)\right)\right]+\mathrm{E}\left[p\left(y-\psi\left(y-d_{t}\left(p, \epsilon_{t}\right)\right)\right)\right]+\mathrm{E}\left[v_{t+1}\left(\psi\left(y-d_{t}\left(p, \epsilon_{t}\right)\right)\right)\right]$,

where the first three terms represent the ordering cost, the expected inventory holding and backlogging (or lost sales penalty) cost and the expected revenue incurred at period $t$ respectively, and the last term is the profit-to-go function. We assume for simplicity that $v_{T+1}(x)=0$, that is, at the end of the planning horizon, no cost is incurred for lost sales and no salvage value is imposed on leftover inventory.

In this subsection, we assume that the ordering cost functions $C_{t}(\cdot)$ are convex, which implies that the marginal purchasing cost increases as one orders more. Under this assumption, the literature mainly focuses on developing conditions under which the so-called base-stock list-price type policy is optimal. In a base-stock list-price policy, at each period there is a base-stock level such that an order is placed to raise the inventory level to the base-stock level and a list price is charged if the initial inventory level is below the base-stock level, otherwise no order is placed and a discount is offered. In addition, the higher the initial inventory level, the deeper the discount. When the ordering cost functions are linear, a base-stock list-price policy is optimal under various technical conditions on demand functions and random perturbations.

When the ordering cost functions are general convex functions, such a simple policy is usually not optimal. However, an extended base-stock list-price policy is optimal under various technical conditions on demand functions and random perturbations. In such a policy, at each period there exists a critical point, say $x^{*}$, such that no order is placed when the initial inventory level $x$ at the beginning of this period is above $x^{*}$, while for $x$ below $x^{*}$, the order-up-to level $y^{*}(x)$ is increasing in $x$; the price $p^{*}\left(y^{*}(x)\right)$ depends on $x$ only through the order-up-to level $y^{*}(x)$ and is decreasing in $x$. Here the critical point, the order quantity and the order-up-to level are usually time dependent. The extended base-stock list-price policy can be refined when the ordering cost functions are convex and piecewise-linear.

A typical approach to prove the optimality of the base-stock list-price type policy is to argue that certain concavity properties on the value functions $v_{t}$ can be preserved under dynamic programming recursions. To illustrate the idea, consider the case in which any unfilled demand is 
backlogged and the expected demands are linear in prices. In this case, single period expected profit functions are concave. If $v_{t+1}(\cdot)$ is concave, then problem $(7)$ has a concave objective function with linear constraints and thus $v_{t}(x)$ remains concave. One can also show that the objective function is supermodular in $(x, y,-p)$ and the feasible set is a lattice. Thus, the optimal order-up-to level $y^{*}(x)$ is increasing in $x$ while the the optimal price $p^{*}\left(y^{*}(x)\right)$ is decreasing in $x$. That is, the optimal policy follows the extended base-stock list-price policy, which reduces to the base-stock list-price policy for linear ordering costs. The same structural policy remains optimal if the demand functions have the forms $d(p) \epsilon_{1}+\epsilon_{2}$ with $\epsilon_{1}$ and $\epsilon_{2}$ being two random perturbations and at each period the expected revenue is a concave function of the expected demand. The idea is to reformulate problem (7) by replacing the price variable with a new variable representing the expected demand (for this purpose we assume that there is a one-to-one correspondence between price and its associated expected demand).

Problem (7) with linear ordering costs and backlogged demand is analyzed in Federgruen \& Heching (1999). They use the mixture demand model $d_{t}\left(p, \epsilon_{t}\right)=d_{1, t}(p) \epsilon_{t}+d_{2, t}(p)$. They further assume that one period expected inventory holding and backlogging cost is jointly convex in inventory level and selling price and illustrate that this assumption is satisfied if $d_{1, t}(p)$ and $d_{2, t}(p)$ are linear in $p$. They prove that a base-stock list-price policy is optimal. Several extensions are discussed. In one extension, price is only allowed to markdown. In this case, again the optimal policy is given by a base stock list price policy with the base stock level dependent on the price of the previous period. They consider another extension in which capacity constraints are imposed for ordering quantities and prove that a modified base stock list price policy is optimal. In such a policy, at each period, when the initial inventory level is below the base stock level, an order would be made to raise the inventory level to the base stock level if possible. Otherwise a full capacity would be ordered. Federgruen \& Heching (1999) further prove that similar structural results can be extended to models with emergency orders (instead of backlogging) and to infinite horizon models under both the discounted profit and long run average profit criteria. However, models with nonzero lead time for ordering impose a significant challenge, for which they propose heuristics.

Federgruen \& Heching (1999) develop an efficient value iteration method to compute optimal policies. Based on data collected from specialty retailer of high end women's apparel, they conduct 
an extensive numerical study to demonstrate the benefits of dynamic pricing strategies over static strategies and the impact of demand uncertainty and price elasticities on optimal policies and their corresponding profits and observe that dynamic pricing strategies may bring significant benefits even in a stationary environment.

Problem (7) with lost sales is more complicated. Indeed, strong technical conditions on both the expected demands and the distributions of the random perturbations are usually needed for the concavity of the single period expected profit. Mills (1962) recognizes the need and the difficulty of extending the single period model in Mills (1959) to multi-period settings and develops a single period approximation.

Miercort (1968) analyzes problem (7) with linear ordering costs. The expected demand is assumed to be decreasing, convex and twice differentiable in price. Under some strong conditions on the demand density functions to ensure the concavity of the single period expected profit, Miercort (1968) proves that a base stock list price policy is optimal.

Ernst (1970) assumes that all costs are linear, demand is additive and linear in price in problem (7). In addition, the distribution of the underlying additive random variable belongs to the class of Pólya frequency functions of order 2 (PF2), which includes common distributions such as uniform, normal and truncated normal as special cases. With additional assumptions on system parameters, the author proves that the optimal policy is unique and follows a base-stock list-price policy ${ }^{8}$. Ernst (1970) extends the results to infinite horizon models, conducts sensitivity analysis of the optimal parameters and compares the optimal policies of the model with uncertain demand and its deterministic counterpart.

Zabel (1972), motivated by observations made from a simulation study in Nevins (1966) on multi-period inventory and pricing models, analyzes a special case of the general model (6) with stationary problem parameters under the assumption that the ordering cost is convex, the inventory holding cost is linear and unsatisfied demand is lost without incurring a penalty cost. The author demonstrates the difficulty of extending the uniqueness properties of optimal solutions from a single period model in Zabel (1970) to multi-period settings. Focusing on an additive demand model in

\footnotetext{
${ }^{8}$ Ernst (1970) pays special attention to the possibility of having negative values derived from additive demand models and illustrates that a list price policy may fail to be optimal if the additive demand model results in negative values at the optimal policy.
} 
which the expected demand is concave in the selling price and the underlying random variable is exponentially or uniformly distributed and imposing some additional technical conditions, Zabel (1972) argues that the profit-to-go function $v_{t}(x)$ is concave and proves that the extended base-stock list-price policy is optimal and in addition the optimal order quantity is decreasing in $x$. The author also makes intra-period comparison of the optimal policy parameters and performs comparative statics analysis. For instance, Zabel (1972) proves that the critical point $x_{t}^{*}$ is increasing over time and the optimal order-up-to level $y_{t}^{*}$, the optimal order quantity $q_{t}^{*}$ and the optimal price $p_{t}^{*}$ are all decreasing in the unit holding cost for $x$ below $x_{t}^{*}$.

Focusing on additive demand, Thowsen (1975) proposes a model similar to Zabel (1972) with linear ordering cost. The author assumes that unsatisfied demand can be partially backlogged which makes the lost sales and full backlogging cases as two extreme cases in a unified model, and a fraction of inventory may deteriorate from one period to the next. Some assumptions on cost and expected demand are also imposed, which essentially require that there is no motive to hold inventory, stock out and backlog demand, the inventory system is profitable and the price constraint will not be binding (i.e., not attain its upper bound). Under these assumptions, Thowsen (1975) identifies conditions under which a base-stock list-price policy is optimal.

As acknowledged by Thowsen (1975), these conditions are hard to verify in general. However, they are satisfied in two settings. The first is the backlogging case with convex inventory holding and backlogging cost and concave expected demand (as a function of price). The second setting is the partial backlogging case as well as the lost sales case with convex inventory holding, linear stock out cost, linear expected demand and a PF2 distribution for the additive random perturbation. In these two settings, the profit-to-go function $v_{t}(x)$ is shown to be concave. The author also allows revenue to be collected several periods after demand occurs or is satisfied (customers still pay the price of the period at which their demand occurs) and proves that the same structural results hold under similar conditions.

Chan et al. (2006) study a multi-period joint pricing and production model under a general, non-stationary stochastic demand function with a discrete menu of prices. In their model, the available production capacity is limited and unmet demand is lost. They also allow discretionary sales, that is, inventory may be set aside to satisfy future demand even if some present demand is lost. They analyze and compare delayed production strategies (in which pricing is determined up 
front while production is determined at the beginning of each period) and delayed pricing strategies (in which production is determined up front while pricing is determined at the beginning of each period). Chan et al. (2006) describe policies and effective heuristics for the strategies based on deterministic approximations. Their computational study illustrates that delayed production is usually better than delayed pricing except sometimes when capacity is tight.

Almost all papers in the inventory and pricing literature assume that the yield is certain. One exception is Li \& Zheng (2006) who analyze a model similar to Federgruen \& Heching (1999) but with additive demand and stochastic proportional yield (i.e., the received quantity equals the order quantity multiplied by a nonnegative random variable). Similar to inventory models with random yield but without pricing decisions (see, for instance, Zipkin, 2000), they prove that the extended base-stock list-price policy is optimal and in addition the optimal order quantity is a decreasing function of the initial inventory level. They further study the operational effects of uncertain yield and prove that, in the single-period case, the critical number is independent of the yield variability, while in the multi-period case, it is higher in a system with uncertain yield than in the one with certain yield. Moreover, the system with uncertain yield always charges a higher price.

Allon \& Zeevi (2009) develop a model that integrates capacity investment (or disinvestment), production (inventory), and price decisions. In their model, at the beginning of each period, a capacity adjustment is first determined, which is then followed by the production and pricing decisions. Demand is additive with expectation linear in price, and unsatisfied demand is backlogged. Inventory holding and backlogging cost is assumed to be convex in leftover inventory level. All other costs, including ordering cost, and capacity investment cost and disinvestment cost, are linear. They prove that the capacity decision follows the so called target interval policy. In such a policy, at each period, there exists two numbers $L$ and $U$ with $L \leq U$ such that the following action is taken: invest and raise capacity to $L$ if the initial capacity level is lower than $L$; disinvest and decrease capacity to $U$ if the initial capacity level is higher than $U$; otherwise keep the current capacity level. In their model, the two numbers $L$ and $U$ may depend on time and initial inventory level. The optimal production and pricing decision follows the modified base-stock list-price policy with the policy parameters dependent on capacity level. They also consider a different case in which inventory cannot be carried over from one period to the next and price can only be marked down. In this case, they prove that the optimal capacity decision takes the form of price dependent target 
inventory policy and the two target parameters are non-increasing in price level of the previous period.

Qi (2010) analyzes an integrated inventory and pricing model with supply capacity uncertainty and linear ordering cost. She illustrates that the base-stock list-price policy is not optimal even under deterministic demand. Assuming additive demand, she proves that in the optimal policy, there exists a reorder point such that an order is placed when the inventory level is below the reorder point, and the optimal order and price are chosen to achieve a constant target safety stock.

Boyaci \& Ozer (2009) consider pricing strategy in acquiring demand information to plan for capacity. They study the joint benefit of acquiring information for capacity planning through advance selling and revenue management of installed capacity through dynamic pricing.

Similar to Chen \& Hu (2008), Chen et al. (2008) incorporate price adjustment costs into an integrated inventory and pricing model but with stochastic demand. Their model is similar to the one analyzed in Federgruen \& Heching (1999), except that at each period, a price adjustment cost is incurred if the current period price is different from the price of the previous period, which may involve a fixed component independent of the magnitude of the price change and a variable component proportional to the magnitude of the price change. Acknowledging the complexity of the general model, Chen et al. (2008) analyze two special cases: a model without fixed ordering cost and fixed price adjustment cost and a model with fixed price adjustment cost and no inventory carryover. For each case, they characterize the structure of the optimal policy.

For the model without fixed ordering cost and fixed price adjustment cost, Chen et al. (2008) prove that the optimal inventory policy follows a base-stock-type policy, in which the base-stock level is a non-increasing function of the price in the previous period, whereas the optimal price follows a target interval policy, in which the two target parameters are non-increasing functions of the initial inventory level. For the model with fixed price-change costs and no inventory carryover, Chen et al. (2008) employ the concepts of $k$-concavity and symmetric $k$-concavity to provide a characterization of the structure of the optimal pricing policy for their model which allows for markdown, markup or bi-directional price changes. For the general problem, they develop an intuitive heuristic policy to manage inventory and set selling prices. Compared with the optimal policy, which is likely to be very complicated, their heuristic policy is amenable to practical implementation. In addition, their numerical study demonstrates that it is quite effective. 
A few works start to incorporate reference price effects into stochastic inventory and pricing models. In this case, the dynamic program (7) has to be augmented to include the reference price as a state variable in addition to the inventory level, which significantly complicates the analysis of the problem. Indeed, the single period expected profit is not a concave function in general. Focusing on additive demand, Gimpl-Heersink (2008) proves that a reference price dependent basestock list-price policy is optimal under general assumptions for a single period setting and under more restrictive assumptions for a two period setting. Their numerical study illustrates that with the presence of reference price effects, the integrated inventory and pricing model brings considerable benefits over a procedure that determines price decisions and ordering decisions sequentially. By introducing a transformation technique, Chen \& Zhang (2009b) prove that a reference pricedependent base-stock policy is optimal in multi-period settings with both additive and multiplicative demands. They also analyze the convergence of the price trajectory in the infinite horizon setting and characterize the limit. Guler et al. (2010) analyzes a similar model where customers are loss-averse or loss-neutral with relative difference reference effects and provide conditions under which a state-dependent order-up-to policy is optimal.

Chen \& Zhang (2010) introduce a stochastic term in reference price evolution dynamics to capture possible modeling errors. They derive an explicit solution for the optimal steady-state price in a continuous time model and illustrate that the optimal steady-state price in the setting with stochastic reference price dynamics is always higher than the corresponding price in the setting without stochasticity.

All the above models assume that inventory is managed at a single location. Federgruen \& Heching (2002) extend their earlier work in Federgruen \& Heching (1999) to a distribution system with a distribution center serving several retailer stores. At the beginning of each period, the distribution center, which does not hold any inventory, will place an order which will then be allocated to the retailer stores after an order lead time and an allocation lead time. At the same time, a single price across all retailer stores is determined. Federgruen \& Heching (2002) present a tractable approximation of the stochastic model, which admits an optimal policy with a simple structure, and carry out an extensive computational study to illustrate the benefits of dynamic pricing strategies under a variety of different system parameters.

A few papers analyze multiple products in the stochastic setting. Zhu \& Thonemann (2009) 
extend the model in Federgruen \& Heching (1999) to the case with two substitutable products in which demand of each product depends linearly on the prices of both products. They prove that the optimal inventory policy behaves similarly to the base-stock policy for the one-product problem: when the starting inventory levels of both products are low, the optimal decision is to order both products; when the starting inventory levels of both products are high, the optimal decision is not to order; when one product has a high starting inventory level and the other has a low starting inventory level, the optimal decision is to order only the product with the low inventory level. In addition, the inventory policy of one period is given by a base stock policy in which the base stock level is non-increasing in the initial inventory level of another product. They also provide a characterization of the optimal pricing and illustrate that in their model, the base-stock list-price policy, which is optimal for the single period model in Federgruen \& Heching (1999), may fail to be optimal.

Ceryan et al. (2009) extend the model in Zhu \& Thonemann (2009) by incorporating both dedicated and flexible production capacities for the two substitutable products. They characterize the structure of the optimal production and pricing decisions and explore the effects of various problem parameters on the optimal policy. Song \& Xue (2007) analyze a model with multiple substitutable products with more general demand functions. They provide a characterization of

the structure of the optimal policy and develop an algorithm to compute it. By developing a preservation result of submodularity property under an optimization operation in two dimensional space, Chen et al. (2010b) characterize the structure of the optimal production and pricing policy for models with either two complementary products or two substitutable products. In the case with two substitutable products, they provide refined structural property and significantly simplify some of the proofs in Zhu \& Thonemann (2009), Ceryan et al. (2009) and Song \& Xue (2007).

\section{Multi-period Models with Concave Ordering Costs}

In the previous subsection we assume that the ordering cost is either convex or linear. However, in some practical settings, the ordering cost can be concave, as a result of economies of scale or incremental discounts provided by suppliers. Such cost structures impose a significant challenge for multi-period models because the value functions $v_{t}(x)$ are not concave anymore and base-stock listprice policies are not optimal either. The literature mainly focuses on developing conditions under 
which an $(s, S, \mathbf{p})$ type policy is optimal when the ordering cost involves a fixed cost component representing economies of scale and a variable cost component. In an $(s, S, \mathbf{p})$ policy, inventory is managed based on an $(s, S)$ policy: if the inventory level at the beginning of period $t$ is below the reorder point, $s_{t}$, an order is placed to raise the inventory level to the order-up-to level, $S_{t}$; otherwise, no order is placed. The selling price of the product depends (not necessarily monotonically) on the initial inventory level at the beginning of the period.

Interestingly, the structural results and the analysis depend heavily on how uncertainty is incorporated into demand models. Consider problem (7) in which the ordering cost includes both a fixed cost independent of the order quantity and a variable cost proportional to the quantity and unsatisfied demand is backlogged. Assume that for any period $t$, the demand function satisfies $d_{t}\left(p_{t}, \epsilon_{t}\right)=\epsilon_{1 t} d_{t}\left(p_{t}\right)+\epsilon_{2 t}$, where the random perturbations, $\epsilon_{t}=\left(\epsilon_{1 t}, \epsilon_{2 t}\right)$, are independent across time satisfying $\epsilon_{1 t} \geq 0, E\left\{\epsilon_{1 t}\right\}=1$ and $E\left\{\epsilon_{2 t}\right\}=0$. Furthermore, the function $d_{t}(p)$ is continuous and strictly decreasing and the expected revenue $d p_{t}(d)$ is concave in the expected demand $d$ at period $t$, where $p_{t}(d)$ is the inverse function of $d_{t}(p)^{9}$.

When the demand process is additive, i.e., $\epsilon_{1 t}=1$, one can show that at an optimal policy, the higher the inventory level at the beginning of time period $t$, the higher the expected inventory level at the end of period $t$. Though the value function $v_{t}(x)$ is not concave, this result allows one to show by induction that $v_{t}(x)$ is a $k$-concave ${ }^{10}$ function of $x$ and the optimality of an $(s, S, \mathbf{p})$ policy follows directly from $k$-concavity.

Unfortunately, the $(s, S, \boldsymbol{p})$ policy is not necessarily optimal for general demand models. To characterize the optimal policy in this case, a new concept, symmetric $k$-concavity ${ }^{11}$ is needed.

\footnotetext{
${ }^{9}$ Since $d=E\left\{d_{t}\left(p_{t}, \epsilon_{t}\right)\right\}=d_{t}\left(p_{t}\right)$, the expected revenue can be represented as $d p_{t}(d)$.

${ }^{10}$ The concept of $k$-convexity is introduced by Scarf (1960) to analyze stochastic inventory models with fixed ordering cost. A definition of $k$-convexity, equivalent to the original one proposed by Scarf, is as follows: a real-valued function $f$ is called $k$-convex for $k \geq 0$, if for any $x_{0} \leq x_{1}$ and $\lambda \in[0,1]$,

$$
f\left((1-\lambda) x_{0}+\lambda x_{1}\right) \leq(1-\lambda) f\left(x_{0}\right)+\lambda f\left(x_{1}\right)+\lambda k
$$

A function $f$ is $k$-concave if $-f$ is $k$-convex.

${ }^{11} \mathrm{~A}$ real-valued function $f$ is called symmetric $k$-convex for $k \geq 0$, if for any $x_{0}, x_{1}$ and $\lambda \in[0,1]$,

$$
f\left((1-\lambda) x_{0}+\lambda x_{1}\right) \leq(1-\lambda) f\left(x_{0}\right)+\lambda f\left(x_{1}\right)+\max \{\lambda, 1-\lambda\} k
$$

A function $f$ is called symmetric $k$-concave if $-f$ is symmetric $k$-convex.
} 
It can be shown that for general demand processes, even though $v_{t}(x)$ may fail to be $k$-concave, it is symmetric $k$-concave and consequently an $(s, S, A, \boldsymbol{p})$ policy is optimal. In such a policy, the optimal inventory strategy at period $t$ is characterized by two parameters $\left(s_{t}, S_{t}\right)$ and a set $A_{t} \in\left[s_{t},\left(s_{t}+S_{t}\right) / 2\right]$, possibly empty depending on the problem instance. When the inventory level $x_{t}$ at the beginning of period $t$ is less than $s_{t}$ or $x_{t} \in A_{t}$, an order of size $S_{t}-x_{t}$ is made. Otherwise, no order is placed. Price depends on the initial inventory level at the beginning of the period.

The above model was first analyzed by Thomas (1974). Although a counterexample is constructed to show that an $(s, S, \boldsymbol{p})$ policy may fail to be optimal when prices are restricted to a discrete set, the author conjectures that an $(s, S, \boldsymbol{p})$ policy is optimal under fairly general conditions if prices in an interval are under consideration.

Chen \& Simchi-Levi (2004a) prove that the $(s, S, \boldsymbol{p})$ policy postulated by Thomas (1974) is indeed optimal when the demand process is additive and construct an example which shows that the value function $v_{t}(x)$ may not be $k$-concave and an $(s, S, \boldsymbol{p})$ policy may fail to be optimal when the demand process is multiplicative. The concept of symmetric $k$-concavity ${ }^{12}$ and the $(s, S, A, \boldsymbol{p})$ policy are introduced in Chen \& Simchi-Levi (2004a) for the multiplicative demand case. Although an $(s, S, A, \boldsymbol{p})$ policy may not be optimal in settings with general demand, surprisingly, Chen \& Simchi-Levi (2004b) show by employing the concept of symmetric $k$-concavity that a stationary $(s, S, \boldsymbol{p})$ policy is optimal for the infinite horizon model under either the discounted profit or the average profit criterion. This optimality result also holds under the average profit criterion if the single period maximum expected profit function, $Q(x)=\max _{p \in[\underline{p}, \bar{p}]} \pi(x, p)$, is quasi-concave, where $\pi(x, p)$ is the single period expected profit for a given inventory level $x$ and price $p$. However, they demonstrate through one example that if prices are restricted to take values from a discrete set, a stationary $(s, S, \boldsymbol{p})$ policy may not be optimal anymore.

Yin \& Rajaram (2007) present a generalization of the model in Chen \& Simchi-Levi (2004a) to allow for Markovian modulated demand. For Chen and Simchi-Levi's infinite horizon model with the average profit criterion, Feng \& Chen (2004) prove that the structure of the optimal policy holds under slightly relaxed demand assumptions and develop an efficient algorithm to find the

\footnotetext{
${ }^{12}$ Building upon the concepts of $k$-convexity and symmetric $k$-convexity, Ye \& Duenyas (2007) propose the so-called $(k, q)$-convexity, which finds application for capacity investment problems with two-sided fixed-capacity adjustment costs (see Ye \& Duenyas, 2007) and stochastic cash balance problems with fixed costs (see Chen \& Simchi-Levi, 2009).
} 
parameters of the optimal policies. On the other hand, Zhang \& Fu (2005) derive sample path derivatives that can be used in a gradient-based algorithm for determining the optimal stationary $(s, S, \mathbf{p})$ policy parameters in a simulation-based optimization procedure.

Focusing on additive demand, Chen et al. (2010c) analyze problem (7) with concave piecewiselinear ordering cost. Under the assumption that the additive random perturbation follows a positive Pólya or uniform distribution, they prove that the value functions $v_{t}(x)$ belong to the class of quasi$k$-concave functions ${ }^{13}$ by establishing a new preservation property of quasi- $k$-concavity, which says that under mild technical conditions, $\max _{d}[\alpha(d)+\beta(y-d)]$ is quasi- $K$-concave if the one-dimensional functions $\alpha(\cdot)$ and $\beta(\cdot)$ are concave and quasi- $K$-concave respectively. Quasi- $k$-concavity of the value functions implies that a generalized $(s, S, \boldsymbol{p})$ policy is optimal. In such a policy, inventory is managed based on a generalized $(s, S)$ policy. That is, there is a sequence of reorder points $s_{i}$ and order-up-to levels $S_{i}$ (both are increasing in $i$ ) such that if the starting inventory level is lower than the reorder point $s_{i}$ but higher than $s_{i+1}$, an order is placed to raise its inventory level to $S_{i}$. The optimal price is set according to the inventory level after replenishment. For the special case with two suppliers, one with only variable cost while the other with both fixed and variable costs, they prove that the (refined) generalized $(s, S, \boldsymbol{p})$ policy is still optimal when the additive random component in the demand function has a strongly unimodal density.

Polatoglu \& Sahin (2000) analyze problem (7) with lost sales. They show that an $(s, S, \boldsymbol{p})$ policy is not optimal in general and provide some conditions for the optimality of an $(s, S, \boldsymbol{p})$ policy, which unfortunately may be hard to verify.

In an attempt to attack lost sales models, Chen et al. (2006) make the following assumptions: first, demand is additive, and in the additive demand process $d(p)+\beta$, the expected demand $d(p)$ is decreasing and concave in $p$, and $3 d^{\prime \prime}+p d^{\prime \prime \prime} \leq 0$ for $p \in[c, \bar{p}]$, where $c$ is the variable ordering cost and $\bar{p}$ is the upper bound imposed on $p$; second, the failure rate function of the underlying random variable $\beta$ with probability density function $f$ and cumulative distribution function $F$, $r(u)=f(u) /(1-F(u))$, satisfies $r^{\prime}(u)+[r(u)]^{2}>0$ for $u$ in the support of $\beta$; third, all cost and demand parameters are stationary; fourth, both the inventory holding cost and the stock out cost

\footnotetext{
${ }^{13} \mathrm{~A}$ one-dimensional function $f$ is quasi- $k$-concave if for any $x_{1} \leq x_{2}$ and $\lambda \in[0,1], f\left((1-\lambda) x_{1}+\lambda x_{2}\right) \geq$ $\min \left\{f\left(x_{1}\right), f\left(x_{2}\right)-k\right\}$. It was introduced by Porteus (1971) to analyze stochastic inventory models with concave ordering costs.
} 
are linear, and the salvage value at the end of the planning horizon is equal to the variable ordering cost. Under these assumptions, they prove that an $(s, S, \mathbf{p})$ policy is optimal for their multi-period model. They also illustrate the difficulty of extending their approach to models with a salvage value different from the variable ordering cost and to models with multiplicative demand.

Huh \& Janakiraman (2008) propose an alternate approach to prove the optimality of an $(s, S, \mathbf{p})$ for problem (6) with stationary cost and demand parameters. In their model, the demand takes a more general form: $d(p, \epsilon)$, where $d(p, \epsilon)$ is monotone and concave in $p$ for almost every value of the underlying uncertainty $\epsilon$ (their demand actually allows for other decisions that may influence demand). They impose two conditions on the single period expected profit function $\pi(x, p)$. Let $y^{*}$ be the maximal point of the single period maximum expected profit $Q(x)$. Their first condition requires that $Q(x)$ is quasi-concave, and their second condition requires that for any $y^{*} \leq y^{1}<y^{2}$ and any given price $p^{2}$, there exists a price $p^{1}$ such that $\pi\left(y^{1}, p^{1}\right) \geq \pi\left(y^{2}, p^{2}\right)$ and $\psi\left(y^{1}-d\left(p^{1}, \epsilon\right)\right) \leq \max \left\{y^{*}, \psi\left(y^{2}-d\left(p^{2}, \epsilon\right)\right)\right\}$ for any realization of $\epsilon$, where $\psi(x)=x$ for the backlogging case and $\psi(x)=\max (x, 0)$ for the lost sales case as defined earlier. Roughly speaking, their first condition implies that the closer the inventory level (after ordering) is to $y^{*}$, the higher profit the system can generate in a single period, and their second condition implies that if one starts with a better inventory position (i.e., an inventory position closer to $y^{*}$ ), one can generate more expected profit at the current period and end up at a better inventory position at the beginning of the following period (i.e., an inventory position closer to $y^{*}$ or smaller than $y^{*}$ so that the inventory level can be raised to $y^{*}$ with one order) simultaneously.

Under the above two conditions, Huh \& Janakiraman (2008) prove that a stationary $(s, S, \mathbf{p})$ policy is optimal for the infinite horizon version of problem (6) under the discounted profit criterion (their approach does not work for the infinite horizon model under the average profit criterion). With a third condition, which requires that for any $y^{2}<y^{1} \leq y^{*}$ and any given price $p^{2}$, there exists a price $p^{1}$ such that $\pi\left(y^{1}, p^{1}\right) \geq \pi\left(y^{2}, p^{2}\right)$ and $\psi\left(y^{1}-d\left(p^{1}, \epsilon\right)\right) \geq \psi\left(y^{2}-d\left(p^{2}, \epsilon\right)\right)$ for any realization of $\epsilon$, they prove that an $(s, S, \mathbf{p})$ policy is optimal for the finite horizon problem (6) with stationary input data. For the backlogging model, they prove that the joint concavity of the single period expected profit $\pi(x, p)$ implies that first two conditions, and present an assumption that gives rise to the three conditions in the additive demand case. For the lost sales model, they prove that the assumptions made in Chen et al. (2006) are sufficient for the three conditions. 
Building upon the approach in Huh \& Janakiraman (2008), Song et al. (2009) analyze problem (7) with stationary input data and multiplicative demand $d(p) \epsilon$. They assume that the expected demand $d(p)$ satisfies the following conditions: first, $d(p)$ is strictly decreasing and has an increasing elasticity $-p d^{\prime}(p) d(p)$; second, $d(p) / d^{\prime}(p)$ is monotone and concave; third, $p+d(p) / d^{\prime}(p)$ is strictly increasing. In addition, the underlying random variable $\epsilon$ has an increasing failure rate. Under these assumptions, they prove that the first two conditions in Huh \& Janakiraman (2008) hold and thus a stationary $(s, S, \mathbf{p})$ policy is optimal for the infinite horizon model under the discounted profit criterion. With additional work, they prove that an $(s, S, A, \mathbf{p})$ policy, optimal for the finite horizon model with backlogging in Chen \& Simchi-Levi (2004a), is also optimal for their finite horizon model with lost sales and stationary input data.

All the above papers assume a risk neutral decision maker. Chen et al. (2007b) propose a general framework for incorporating risk aversion in multi-period inventory (and pricing) models, while Chen \& Sun (2007) consider a corresponding infinite horizon model. They show that the structure of the optimal policy for a decision maker with additive exponential utility functions is almost identical to the structure of the optimal risk neutral inventory (and pricing) policies, and demonstrate computationally that the optimal policy is relatively insensitive to small changes in the decision maker's level of risk aversion. Interestingly, since the additivity property of the expectation operator cannot be extended to risk averse setting (involving certainty equivalent operator), the proof approaches in Chen \& Simchi-Levi (2004b) and Huh \& Janakiraman (2008), built upon additivity of the expectation operator, may not be extended to analyze their infinite horizon risk averse model and thus they take a different approach to prove the optimality of the stationary $(s, S, \mathbf{p})$ policy.

Several papers analyze continuous review models with fixed ordering cost. Under the unit demand assumption (i.e., each arrival requests a single unit item), Feng \& Chen (2002) prove that the optimal policy has a simple structure under the infinite horizon long run average profit criterion. Chen \& Simchi-Levi (2006) subsequently generalize their model and results to allow for more general demand functions and prove that an $(s, S, \mathbf{p})$ policy and a stationary $(s, S, \mathbf{p})$ are optimal respectively for the finite horizon case and the infinite horizon case under both the discounted profit and average profit criteria. In the infinite horizon case with average profit criterion, Chen \& Simchi-Levi (2006) prove that the optimal price is a unimodal function of the inventory level. 
Finally, for the same model, Chao \& Zhou (2006) develop efficient computational procedures to find the parameters of the optimal policy.

It is worthwhile to mention several other papers whose focus is not on characterizing the structure of optimal policies. Chen et al. (2010a) present an inventory and pricing model with demand modeled as a diffusion process and quantify the profit improvement of dynamic pricing over static pricing. Other related work includes Lodish (1980) who develops an integrated inventory and pricing model and Subrahmanyan \& Shoemaker (1996) who develop an integrated inventory and pricing model with demand learning through Bayesian updating. Without focusing on the structure of the optimal policies, both papers propose to solve their models by brute force backward dynamic programming. Additional papers on demand learning include Petruzzi \& Dada (2002), Aviv \& Pazgal (2005) and Zhang \& Chen (2006).

\section{Supply Chain Competition, Coordination and Cooperation}

The research on decentralized supply chain systems received significant amount of attention in the past decade and remains an active area. One stream of this research employs concepts and methodologies from noncooperative game theory to analyze equilibrium behavior of the supply chain and supply chain coordination. As the literature on these topics is rapidly growing, we do not intend to provide a comprehensive review. Instead, our focus is mainly on decentralized supply chain models (mostly with demand uncertainty) that are either built upon or closely related to the integrated inventory and pricing models reviewed in the previous sections. For more comprehensive recent surveys on decentralized supply chain models, we refer to Cachon (2003) and several chapters in Tayur et al. (1998). We also refer to Kaya \& Ozer (2010) in this handbook for a tutorial on supply chain contracts. Another, more recent and less extensive stream of research employs concepts and methodologies from cooperative game theory to analyze the stability and formation of coalitions in supply chain settings. We review several papers on this stream in which inventory and pricing decisions play an important role.

Levitan \& Shubik (1971) provide a duopoly model with two symmetric firms competing on inventory and price. Even though a pure strategy Nash equilibrium may not exist in a deterministic model with capacity constraints, they illustrate through an example that it may be restored when 
demand is stochastic and inventory carrying cost is introduced. Since they model explicitly priceouts (i.e., a firm is driven out of the market) and customer switching in case of stock-outs, their demand model is very complicated, which prevents them from deriving conditions for the existence of a pure strategy Nash equilibrium.

Marvel \& Peck (1995) consider a single period model consisting of a manufacturer and a retailer who orders from the manufacturer and makes a pricing decision. Demand is characterized by two independent random variables: the number of customers and customers' valuation of the product. A customer with a valuation no less than the retailer's price will purchase one unit. They illustrate that the attractiveness of a return policy to the manufacturer depends crucially on the nature of the demand uncertainty. Specifically, return policies benefit the manufacturer if the only uncertainty is over the number of customers. On the other hand, it would induce retailer prices too high to be beneficial for the manufacturer if the only uncertainty is over customers' valuation.

Kandel (1996) studies the allocation of responsibility of unsold inventories in a setting with a manufacturer and a retailer. The author demonstrates that contract choice may depend on (among several other factors) whether the selling price is a decision variable and who is responsible for making the pricing decision. For instance, when the retailer decides the selling price, it is possible to show that in cases with sufficiently high price elasticity, the manufacturer prefers no return policy, while the opposite is true when the price elasticity is not too high.

Emmons \& Gilbert (1998) consider a similar setting but assume that the retailer faces a (stochastic) multiplicative price-dependent demand. They illustrate that under the assumption of a linear expected demand and uniformly distributed multiplicative random perturbation, there exists a return policy which leads to higher profits for both the manufacturer and the retailer.

Chen et al. (2007a) study a setting in which the manufacturer provides a rebate to end customers of the retailer. Building upon the multiplicative demand model with an iso-price-elastic expected demand, they characterize the impact of a manufacturer rebate on the expected profits of both the manufacturer and the retailer. They show that unless all of the customers claim the rebate, the rebate always benefits the manufacturer, while an "instant rebate", where every customer redeems the rebate on the spot, does not necessarily benefit the manufacturer. 
Granot \& Yin (2005) analyze a model similar to the one in Emmons \& Gilbert (1998). They show that for a zero salvage value and some expected demand functions, return policies may not benefit the manufacturer. They also investigate the impact of return policies on the supply chain. In a similar setting, Granot \& Yin (2008) analyze the effect of price and order postponement on the equilibrium values of the contract parameters and profits, while Granot \& Yin (2007) allow the manufacturer and the retailer to commit to contract parameters (wholesale price, retail price, buy back price and order quantity) sequentially and alternately, and investigate its effect on the equilibrium profits of the channel and its members.

Cachon \& Lariviere (2005) discuss different contracts in single period supply chain settings involving a supplier and a single retailer or competing retailers facing price-dependent demand among many other things. In the case with a single price-setting retailer, they point out that many contracts that coordinate supply chains with a price-fixed retailer may fail to coordinate the supply chain. However, the revenue sharing contract (in which the supplier requires a percentage of realized revenue from the retailer) continues to coordinate the supply chain and is equivalent to the linear price-discount sharing scheme proposed by Bernstein \& Federgruen (2005).

Li \& Atkins (2002) study the coordination of production and marketing within a firm. Assuming linear additive demand, they make the following observations under linear transfer price: (a) if production commits to a service level ${ }^{14}$ instead of an inventory level, both production and marketing are better off; (b) the same improvement can be achieved if marketing is at a dominant position (in the sense that it is leader in a Stackelberg game between production and marketing). In addition, they propose to coordinate the firm through a quantity discount contract coupled with a buy back policy.

Bernstein \& Federgruen (2005) analyze a decentralized supply chain system with a single supplier serving multiple retailers in a single period setting. Each retailer faces a single period problem similar to the ones presented in the single period stochastic models subsection with lost sales and multiplicative demand. They consider both the noncompeting retailer case in which each retailer's demand depends only on its own price and the competing retailer case in which the retailers offer substitutable products and thus each retailer's demand depends on its own price

\footnotetext{
${ }^{14}$ If demand is given by $d(p)+\epsilon$ and the order quantity is $y$, Li \& Atkins (2002) use $z=y-d(p)$ to measure service level. Also recall that $z$ is referred to as the safety stock factor in Petruzzi \& Dada (1999).
} 
and the prices of all other retailers. Since they assume linear production cost for the supplier, it is clear that in the first case, the noncompeting retailers are independent of each other and the qualitative results for a supply chain with a supplier and a retailer can be directly extended to the case with multiple noncompeting retailers. In their second case with competing retailers, Bernstein \& Federgruen (2005) assume that the logarithm of a retailer's expected demand function has increasing differences in its own price and each of the prices of the other retailers. Under this assumption, the retailer game when wholesale prices and buy-back rates are constant belongs to a class of well-understood games, the log-supermodular game, and thus admits a pure strategy Nash equilibrium. In both cases, Bernstein \& Federgruen (2005) propose contracts that coordinate the supply chain, i.e., the expected profit of the decentralized system under the contract is the same as the maximum expected profit achievable in a centralized supply chain.

Ray et al. (2005) study centralized and decentralized supply chains in which a retailer, facing arriving customers with an additive price-dependent rate, makes replenishment from a distributor following a local base-stock policy, who in turn orders from an outside manufacturer again using a local base-stock policy. In their decentralized model, the distributor and the retailer play a Stackelberg game with the distributor as the leader setting its wholesale price and base-stock level. Ray et al. (2005) investigate the impact of price sensitivity, demand uncertainty and delivery time variability on the decisions of the distributor and the retailer under both the centralized and decentralized settings.

Wang et al. (2004) analyze consignment contracts with revenue sharing in a single period setting in which the manufacturer and the retailer play a Stackelberg game but with the retailer as the leader in setting the percentage of realized revenues shared with the manufacturer. The manufacturer will then decide on the selling price and delivery quantity for the retailer and maintain the ownership of the stock. Wang (2006) extends the model in Wang et al. (2004) to a setting with multiple manufacturers producing and selling perfectly complementary products (i.e. products that are always sold together) to the market through a common retailer under an identical consignment contract with revenue sharing.

Kirman \& Sobel (1974) appear to be the first to analyze a periodic review infinite horizon model with competing firms facing stochastic demands. They assume that unsatisfied demand is backlogged and all costs (ordering, inventory holding, backlogging) are linear. Their principal 
result is to show that a pure strategy Nash equilibrium with a stationary base-stock level ${ }^{15}$ for the infinite horizon game is specified by a pure strategy Nash equilibrium of a corresponding single stage game ${ }^{16}$. The existence of this equilibrium is guaranteed under certain technical conditions imposed on the expected single period expected profit (as a function of the order quantity and the selling price). Bernstein \& Federgruen (2004a) consider a periodic review infinite horizon model with competing retailers similar to Kirman \& Sobel (1974) with multiplicative demand. Under some technical conditions, they show that the corresponding single stage game is log-supermodular and thus a pure strategy Nash equilibrium exists, which in turn specifies a pure strategy Nash equilibrium of the infinite horizon game in which each retailer adopts a stationary base-stock policy together with a stationary price. They also perform comparative statics analysis regarding the impact of several system parameters on the equilibrium strategy and its associated expected profits.

Bernstein \& Federgruen (2004b) develop a periodic review infinite horizon general equilibrium model with price and service competition in which service level of a firm is measured by its fill rate - the fraction of demand filled from on-hand inventory. They assume that demand of each firm is multiplicative and propose various forms of the expected demand as a function of firms' prices and service levels. Three different competition scenarios are analyzed: (a) price competition with exogenous service levels; (b) simultaneous price and service-level competition; (c) two stage competition in which each firm first sets a service level and then chooses a dynamic pricing and inventory policy. The supply chain coordination under price and service competition is addressed in Bernstein \& Federgruen (2007).

Competition models incorporating economies of scale in the operational costs and time dependent demand functions and cost parameters are limited and challenging. Federgruen \& Meissner (2009) study a setting with multiple competing retailers each of which faces a joint inventory and static pricing problem similar to the one in Kunreuther \& Schrage (1973). At each period, each

\footnotetext{
${ }^{15}$ Kirman \& Sobel (1974) do not articulate the pricing strategies of the firms, which presumably are mixed strategies. Bernstein \& Federgruen (2004a) comment that Kirman and Sobel's characterization of the mixed strategy equilibrium requires a modification.

${ }^{16}$ Kirman \& Sobel (1974) argue that all their results can be extended to the lost sales model by observing that a lost sales model can be equivalently converted to a backlogging model by granting a credit for each backlogged unit. This observation unfortunately is not true when prices are also decisions variables.
} 
retailer's demand is a function of the prices of all retailers set at the beginning of the planning horizon. They develop close approximations to the profit functions. In addition, they prove that under mild conditions the price competition game under these approximate profit functions has a pure strategy Nash equilibrium and with slightly stronger conditions, it has a unique equilibrium, which is the limit of the tatônnement scheme starting from any initial price vector.

Several papers consider centralized and decentralized two-echelon distribution systems with a supplier serving a network of retailers, each of which faces an EOQ-type of setting with a constant price-dependent demand rate (i.e., each retailer places orders to satisfy a constant and continuous demand flow while balancing fixed ordering costs and inventory holding costs). Chen et al. (2001b) develop efficient algorithms to determine optimal pricing and replenishment strategies for different channel structures. Boyacı \& Gallego (2002) study the problem of coordinating pricing and inventory replenishment policies in a supply chain consisting of one wholesaler and one or more noncompeting retailers with deterministic price-sensitive demand and show that an optimal solution for the centralized system can be interpreted as consignment selling. Chen et al. (2001a) and Bernstein \& Federgruen (2003) propose coordination mechanisms for the decentralized systems with noncompeting retailers and competing retailers respectively. Bernstein et al. (2006) characterize supply chain settings in which perfect coordination can be achieved with simple wholesale pricing schemes and apply their results to decentralized distribution systems. For more EOQ-type supply chain models, see Yano \& Gilbert (2003).

All the above papers employ the concepts and methodologies from noncooperative game theory, which basically assume that no binding agreements can be enforced. However, there are numerous situations in which binding agreements, once reached, can be costlessly implemented. Another branch of game theory, cooperative game theory, provides concepts and tools to analyze these binding agreements and started to receive the attention from the operations management community. We review two recent papers which apply concepts and methodologies from cooperative game theory to study supply chain models involving inventory and pricing decisions. For a comprehensive review of the applications of cooperative game theory to supply chain settings, we refer to Nagarajan \& Sosic (2008).

Chen (2009) considers a distribution system consisting of a set of noncompeting retailers, who sell a single product. The demand of each retailer depends on its own selling price and a common 
random variable representing the market condition, referred to as a market signal. To take advantage of economies of scale and risk pooling effects, the retailers may form a coalition by placing joint orders before the realization of the market signal and allocating inventory among themselves after the market signal is revealed. Given retailers' interest in inventory centralization, it is critical that they allocate the cost or share the benefit in such a way that no set of retailers gains more by deviating from the cooperation. Such cooperatively achieved allocations are called core allocations in cooperative game theory. The existence of core allocations implies that the grand coalition (i.e., the coalition with all retailers) is stable ${ }^{17}$. Chen (2009) shows that inventory centralization games with price dependent demand have nonempty cores under very general assumptions regarding ordering costs. Specifically, under the assumption that the ordering cost follows a general quantity discount (which includes several commonly used discounts: incremental discounts, all-units discounts and the less-than-truckload volume discount), he proves that an inventory centralization game in which all retailers share a single common warehouse has a nonempty core when (a) the retailers pricing decisions are made after the revelation of the market signal, or (b) the retailers have identical cost parameters and their pricing decisions are made before the revelation of the market signal.

The model in Chen (2009) assumes each retailer's demand is independent of other retailers' prices. When this assumption is relaxed, it becomes tricky to define core allocations as the value of a coalition depends on pricing decisions of retailers not in the coalition. In fact, in this case, the retailers may prefer to form different price-setting cartels. Nagarajan \& Sosic (2007) study the dynamic alliance formation process for a system with multiple symmetric retailers competing on (price) substitutable products. In their model, the retailers, facing a deterministic (or stochastic additive) linear demand, make two stage decisions. In the first stage, the retailers form coalitions. In the second stage, retailers in the same coalition agree on a single price to compete with the prices of other coalitions. In the case with demand uncertainty, retailers in the same coalition are assumed to make their own inventory decisions independently after the prices are set.

Nagarajan \& Sosic (2007) apply two solution concepts from cooperative game theory, the largest consistent set and the equilibrium process of coalition formation, to study the stability of

\footnotetext{
${ }^{17}$ Chen \& Zhang $(2007,2009 a)$ study core allocations of distribution systems respectively in a similar single period stochastic setting and in a deterministic multi-period setting. Both papers assume concave ordering costs but do not consider pricing decisions.
} 
coalition structures in a farsighted sense. They identify conditions under which certain coalition structures are farsighted stable and investigate the impact of the market size, demand variability, cost parameters and degree of substitutability on market structures.

\section{Conclusions and Future Research}

As can be seen from the above survey, many papers on integrated inventory and pricing models appeared in the past few years. Some notable progress includes: (a) the conditions for the optimality of an $(s, S, \boldsymbol{p})$ policy for multi-period stochastic models with concave ordering costs have been relaxed; (b) researchers started to explore integrated inventory and pricing models incorporating customer behavior; (c) models that explicitly incorporate price adjustment costs started to receive attention; (d) research on decentralized supply chain models with price dependent external demand grew rapidly; (e) models analyzing the stability and formation of coalitions from the perspective of cooperative game theory emerged.

Yet many important problems remain to be explored. First, our understanding of integrated inventory and pricing models incorporating customer behavior is still very limited. The reference price model presented earlier can be immediately integrated with existing inventory and pricing models, which unfortunately aggravates the difficulty of the already complicated models. However, given its strong empirical and theoretical support, it is definitely valuable to incorporate it into production/inventory models. Of course, the reference price model only provides one illustrative example. We believe that many more customers behavior models will be built and incorporated into operational models.

Second, it remains a significant challenge to incorporate lead time into stochastic models. Indeed, the zero lead time assumption is required for all the multi-period models reviewed here, which severely limits their use and our understanding of the interaction between inventory and pricing decisions in more general settings such as multi-echelon systems, assembly systems and distribution systems. By contrast for standard stochastic inventory control problems with backlogging, structural results of the optimal policy for models with zero lead time can generally be extended to models with deterministic lead time. The idea is to transfer a model with positive lead time to one with a similar structure, but zero lead time (see Scarf, 1960). Unfortunately, this technique 
is not valid for inventory and pricing models with positive lead time because in this case, the two decisions, the ordering decision and the pricing decision, will take effect at different times.

Third, more research on multi-product models is needed. In inventory models without pricing, different products are linked together through joint fixed ordering costs and/or shared capacity and/or correlated demand. In integrated inventory and pricing models, they may also be linked together through cross price elasticities. These linkages greatly enrich the applicability of models. On the other hand, they also impose enormous challenges in terms of collecting data as well as analyzing and solving multi-period models.

Fourth, the structure of the optimal policy for the stochastic integrated inventory and pricing model with both fixed ordering cost and fixed price adjustment cost is still open. Indeed, in this case, unlike the model analyzed in Chen \& Simchi-Levi (2004a), inventory level alone is not enough to specify the profit-to-go function and the previous period price has to be incorporated. As a result, we end up with a challenging dynamic program with two dimensional state space for which the concept of symmetric $k$-convexity, important for integrated inventory and pricing models without price adjustment cost or without inventory carryover, is unlikely to be applicable. Yet, we believe that extensions of this concept (symmetric $k$-convexity) will prove powerful for two or higher dimensional dynamic programs. In fact, a deep understanding of this problem will not only allow the analysis of general integrated inventory and pricing models with costly price adjustment but also shed new lights on another class of classical inventory models, namely the stochastic joint replenishment problems, given the similarities of their structures. It is worthwhile pointing out that the structure of the optimal policy of stochastic joint replenishment problems is essentially unknown despite several decades of intensive research on inventory models.

Fifth, incorporating insights derived from decentralized supply chain models into decision making may provide tremendous values in improving how organizations interact. Unfortunately, limited research has been conducted on multi-period competition models. This is not surprising given the complexity of inventory and pricing models. But, the tremendous progress that has been achieved in the last few years will hopefully lead to new models that capture competition.

Finally, there are still significant gaps between academic research and industrial practice. Several vendors such as JDA, Oracle and SAS provide both pricing optimization and inventory management modules. However, we are not aware of any decision support system that truly inte- 
grates production/inventory and pricing decisions despite their potential impacts. Several factors may contribute to this:

- The lack of efficient algorithms for general, multi-period, multi-product models, slow the development of decision support systems. This is even true for single-product models because of the distinctive features of these models: multi-period, uncertainty and economies of scale. Indeed, even in deterministic settings, incorporating pricing decisions significantly increases the computational complexity relative to pure inventory models.

- The existence of organizational barriers. That is, inventory decisions are typically the responsibility of manufacturing, logistics or supply chain executives whereas pricing is controlled by finance, sales and marketing;

- The lack of empirical data to help companies identify the appropriate demand-price function. In some industries, such data can be collected through online experiments. In others, it is done through customer market survey conducted before new products are released. Our experience is that retailers typically apply multiplicative demand models while the automotive industry applies linear, additive, demand models;

- Finally, the lack of experience in identifying when deterministic models are more appropriate than stochastic models and vice versa.

Thus, while significant progress has been made on the integration of pricing and inventory management, enormous challenges and opportunities remain.

Acknowledgements: The authors thank the editors of this handbook, Peng Hu and Yuhan Zhang for helpful suggestions and comments. The first author is partly supported by NSF Grants CMMI0653909, CMMI-0926845 ARRA and CMMI-1030923. The second author is partly supported by ONR Contracts N00014-95-1-0232 and N00014-01-1-0146; NSF Contracts DMI-0085683 and DMI0245352; NASA interplanetary supply chain and logistics architectures project; and NSF Contract CMMI-0758069. 


\section{References}

V. Agrawal \& S. Seshadri (2000). 'Impact of Uncertainty and Risk Aversion on Price and Order Quantity in the Newsvendor Problem'. Manufacturing \& Service Operations Management 2(4):410-423.

H. Ahn, et al. (2007). 'Pricing and Manufacturing Decisions When Demand Is a Function of Prices in Multiple Periods'. Operations Research 55(6):1039-1057.

R. Ahuja, et al. (1993). Network flows: theory, algorithms, and applications. Prentice Hall, New Jersey.

G. Allon \& A. Zeevi (2009). 'On the Relationship between Price and Capacity Decisions in Inventory Systems with Stochastic Demand'. Production and Operations Management, forthcoming .

Y. Aviv \& A. Pazgal (2005). 'A partially observed Markov decision process for dynamic pricing'. Management Science 51(9):1400-1416.

G. Aydin \& E. Porteus (2008). 'Joint Inventory and Pricing Decisions for an Assortment'. Operations Research 56(5):1247-1255.

F. Bernstein, et al. (2006). 'Coordinating Supply Chains with Simple Pricing Schemes: The Role of Vendor-Managed Inventories'. Management Science 52(10):1483-1492.

F. Bernstein \& A. Federgruen (2003). 'Pricing and Replenishment Strategies in a Distribution System with Competing Retailers'. Operations Research 51(3):409-426.

F. Bernstein \& A. Federgruen (2004a). 'Dynamic inventory and pricing models for competing retailers'. Naval Research Logistics 51(2):258-274.

F. Bernstein \& A. Federgruen (2004b). 'A General Equilibrium Model for Industries with Price and Service Competition'. Operations Research 52(6):868-886.

F. Bernstein \& A. Federgruen (2005). 'Decentralized Supply Chains with Competing Retailers Under Demand Uncertainty'. Management Science 51(1):18-29.

F. Bernstein \& A. Federgruen (2007). 'Coordination Mechanisms for Supply Chains Under Price and Service Competition'. Manufacturing \& Service Operations Management 9(3):242-262. 
S. Biller, et al. (2005). 'Dynamic Pricing and the Direct-to-Customer Model in the Automotive Industry'. Electronic Commerce Research 5(2):309-334.

E. Bish \& Q. Wang (2004). 'Optimal Investment Strategies for Flexible Resources, Considering Pricing and Correlated Demands'. Operations Research 52(6):954-964.

T. Boyacı \& G. Gallego (2002). 'Coordinating pricing and inventory replenishment policies for one wholesaler and one or more geographically dispersed retailers'. International Journal of Production Economics 77(2):95-111.

T. Boyaci \& O. Ozer (2009). 'Information Acquisition for Capacity Planning via Pricing and Advance Selling; When to Stop and Act?'. Operations Research pp. 1328-1349.

G. Cachon (2003). 'Supply Chain Coordination with Contracts'. Hankbooks in Operations Research and Management Science 11:229-340.

G. Cachon \& M. Lariviere (2005). 'Supply Chain Coordination with Revenue-Sharing Contracts: Strengths and Limitations'. Management Science 51(1):30-44.

O. Ceryan, et al. (2009). 'Managing Demand and Supply for Multiple Products Through Dynamic Pricing and Capacity Flexibility'. Working paper .

L. Chan, et al. (2004). 'Coordination of Pricing and Inventory Decisions: A Survey and Classification'. Handbook of Quantitative Supply Chain Analysis: Modeling in the E-Business Era pp. 335-392.

L. Chan, et al. (2006). 'Pricing, production, and inventory policies for manufacturing with stochastic demand and discretionary sales'. Manufacturing \& Service Operations Management 8(2):149168.

X. Chao \& S. Zhou (2006). 'Joint inventory-and-pricing strategy for a stochastic continuous-review system'. IIE Transactions 38(5):401-408.

F. Chen, et al. (2001a). 'Coordination Mechanisms for a Distribution System with One Supplier and Multiple Retailers'. Management Science 47(5):693-708.

F. Chen, et al. (2001b). 'Near-Optimal Pricing and Replenishment Strategies for a Retail/Distribution System'. Operations Research 49(6):839-853. 
H. Chen, et al. (2010a). 'On the Benefit of Inventory-Based Dynamic Pricing Strategies'. Production and Operations Management 19:249-260.

X. Chen (2009). 'Inventory Centralization Games with Price-Dependent Demand and Quantity Discount'. Operations Research 57(6):1394-1406.

X. Chen, et al. (2010b). 'Preservation of Submodularity under Infimal Convolution in Two Dimensional Space and its Applications'. Working paper .

X. Chen \& P. Hu (2008). 'Coordinated Pricing and Inventory Management with Deterministic Demand and Costly Price Adjustment'. Working paper .

X. Chen, et al. (2009a). 'Coordinated Pricing and Inventory Management with Reference Price Effect and Deterministic Demand'. Working paper .

X. Chen, et al. (2007a). 'The Impact of Manufacturer Rebates on Supply Chain Profits'. Naval Research Logistics 54:667-680.

X. Chen, et al. (2007b). 'Risk Aversion in Inventory Management'. Operations Research 55(5):828842.

X. Chen \& D. Simchi-Levi (2004a). 'Coordinating Inventory Control and Pricing Strategies with Random Demand and Fixed Ordering Cost: The Finite Horizon Case'. Operations Research 52(6):887-896.

X. Chen \& D. Simchi-Levi (2004b). 'Coordinating Inventory Control and Pricing Strategies with Random Demand and Fixed Ordering Cost: The Infinite Horizon Case'. Mathematics of Operations Research 29(3):698-723.

X. Chen \& D. Simchi-Levi (2006). 'Coordinating inventory control and pricing strategies: The continuous review model'. Operations Research Letters 34(3):323-332.

X. Chen \& D. Simchi-Levi (2009). 'A New Approach for the Stochastic Cash Balance Problem with Fixed Costs'. Probability in the Engineering and Informational Sciences 23:545-562.

X. Chen \& P. Sun (2007). 'Optimal Structural Policies for Ambiguity and Risk Averse Inventory and Pricing Models'. Working paper . 
X. Chen \& J. Zhang (2007). 'Duality Approaches to Economic Lot-Sizing Games'. Working paper

X. Chen \& J. Zhang (2009a). 'A Stochastic Programming Duality Approach to Inventory Centralization Games'. Operations Research 57(4):840-851.

X. Chen \& Y. Zhang (2009b). 'Stochastic Inventory Models with Reference Price Effects'. Working paper, University of Illinois .

X. Chen \& Y. Zhang (2010). 'Dynamic Pricing under Stochastic Reference Price Effect'. Working Paper .

X. Chen, et al. (2010c). 'Preservation of Quasi-K-Concavity and Its Application to Joint InventoryPricing Models with Concave Ordering Costs'. Operations Research 58(4-Part-1):1012-1016.

X. Chen, et al. (2008). 'Integration of inventory and pricing decisions with costly price adjustments'. $W$ orking paper .

Y. Chen, et al. (2006). 'Optimal pricing and inventory control policy in periodic-review systems with fixed ordering cost and lost sales'. Naval Research Logistics 53(2):117-136.

Y. Chen, et al. (2009b). 'A Risk-Averse Newsvendor Model Under CVaR Decision Criterion'. Operations Research 57(4):1040-1044.

J. Chod \& N. Rudi (2005). 'Resource Flexibility with Responsive Pricing'. Operations Research 53(3):532-548.

J. Dana \& N. Petruzzi (2001). 'Note: The Newsvendor Model with Endogenous Demand'. Management Science 47(11):1488-1497.

S. Deng \& C. Yano (2006). 'Joint Production and Pricing Decisions with Setup Costs and Capacity Constraints'. Management Science 52:741-756.

J. Eliashberg \& R. Steinberg (1991). 'Marketing-production Joint Decision Making'. J. Eliashberg, J. D. Lilien, eds. Management Science in Marketing, Volume 5 of Handbooks in Operations Research and Management Science . 
W. Elmaghraby \& P. Keskinocak (2003). 'Dynamic Pricing in the Presence of Inventory Considerations: Research Overview, Current Practices, and Future Directions'. Management Science 49:1287-1309.

H. Emmons \& S. M. Gilbert (1998). 'Note. the Role of Returns Policies in Pricing and Inventory Decisions for Catalogue Goods'. Management Science 44(2):276-283.

R. Ernst (1970). A Linear Inventory Model with a Monopoly Firm. Ph.D. thesis, University of California, Berkeley.

A. Federgruen \& A. Heching (1999). 'Combined Pricing and Inventory Control under Uncertainty'. Operations Research 47:454-475.

A. Federgruen \& A. Heching (2002). 'Multilocation Combined Pricing and Inventory Control'. Manufacturing \& Service Operations Management 4(4):275-295.

A. Federgruen \& J. Meissner (2009). 'Competition under time-varying demands and dynamic lot sizing costs'. Naval Research Logistics 56:57-73.

Y. Feng \& Y. Chen (2002). 'Joint pricing and inventory control with setup costs and demand uncertainty'. Working paper .

Y. Feng \& Y. Chen (2004). 'Optimality and optimization of a joint pricing and inventory-control policy for a periodic-review system'. Working paper .

G. Fibich, et al. (2003). 'Explicit Solutions of Optimization Models and Differential Games with Nonsmooth (Asymmetric) Reference-Price Effects'. Operations Research 51(5):721-734.

M. Florian, et al. (1980). 'Deterministic Production Planning: Algorithms and Complexity'. Management Science 26(7):669-679.

M. Florian \& K. Morton (1971). 'Deterministic Production Planning with Concave Costs and Capacity Constraints'. Management Science 18(1):12-20.

J. Geunes, et al. (2009). 'Capacitated Procurement Planning with Price-Sensitive Demand and General Concave Revenue Functions'. European Journal of Operational Research 194(2):390405. 
J. Geunes, et al. (2006). 'Requirements Planning with Pricing and Order Selection Flexibility'. Operations Research 54:394-401.

S. M. Gilbert (1999). 'Coordination of pricing and multi-period production for constant priced goods'. European Journal of Operational Research 114(2):330-337.

S. M. Gilbert (2000). 'Coordination of Pricing and Multiple-Period Production across Multiple Constant Priced Goods'. Management Science 46:1602-1616.

D. Gimpl-Heersink (2008). Joint Pricing and Inventory Control under Reference Price Effects. Ph.D. thesis, University of Economics.

D. Granot \& S. Yin (2005). 'On the Effectiveness of Returns Policies in the Price-Dependent Newsvendor Model'. Naval Research Logistics 52(8):765-779.

D. Granot \& S. Yin (2007). 'On sequential commitment in the price-dependent newsvendor model'. European Journal of Operational Research 177(2):939-968.

D. Granot \& S. Yin (2008). 'Price and Order Postponement in a Decentralized Newsvendor Model with Multiplicative and Price-Dependent Demand'. Operations Research 56(1):121-139.

E. Greenleaf (1995). 'The Impact of Reference Price Effects on the Profitability of Price Promotions'. Marketing Science 14:82-82.

M. Guler, et al. (2010). 'Joint Inventory and Pricing Decisions with Reference Effects'. Working Paper .

J. Hall, et al. (2003). 'A Category Management Model of Retailer Dynamic Pricing and Ordering Decisions: Normative and Empirical Analysis'. Working Paper .

A. Hempenius (1970). Monopoly with Random Demand. Universitaire Pers Rotterdam.

W. T. Huh \& G. Janakiraman (2008). '(s, S) Optimality in Joint Inventory-Pricing Control: An Alternate Approach'. Operations Research 56:783-790.

D. Kahneman \& A. Tversky (1979). 'Prospect Theory: An Analysis of Decision under Risk'. Econometrica 47(2):263-91. 
E. Kandel (1996). 'Right to Return, The'. Journal of Law and Economics 39:329-356.

S. Karlin \& C. Carr (1962). 'Prices and optimal inventory policy'. Studies in Applied Probability and Management Science pp. 159-172.

Kaya \& O. Ozer (2010). 'xxxx'. this handbook .

A. Kirman \& M. Sobel (1974). 'Dynamic oligopoly with inventories'. Econometrica 42(2):279-287.

A. Kocabiykoglu \& I. Popescu (2009). 'An Elasticity Perspective on the Newsvendor with Price Sensitive Demand'. Operations Research, forthcoming .

P. Kopalle, et al. (1996). 'Asymmetric Reference Price Effects and Dynamic Pricing Policies'. Marketing Science 15:60-85.

H. Kunreuther \& L. Schrage (1973). 'Joint Pricing and Inventory Decisions for Constant Priced Items'. Management Science 19(7):732-738.

A. H.-L. Lau \& H.-S. Lau (1988). 'The Newsboy Problem With Price-Dependent Demand Distribution'. IIE Transactions 20:168-175.

H. E. Leland (1972). 'Theory of the Firm Facing Uncertain Demand'. The American Economic Review 62(3):278-291.

R. Levitan \& M. Shubik (1971). 'Price Variation Duopoly with Differentiated Products and Random Demand'. Journal of Economic Theory 3:23-39.

D. Levy, et al. (1997). 'The Magnitude of Menu Costs: Direct Evidence from Large U.S. Supermarket Chains'. Quarterly Journal of Economics 112:791-825.

Q. Li \& D. Atkins (2002). 'Coordinating Replenishment and Pricing in a Firm'. Manufacturing \& Service Operations Management 4(4):241-257.

Q. Li \& S. Zheng (2006). 'Joint Inventory Replenishment and Pricing Control for Systems with Uncertain Yield and Demand'. Operations Research 54(4):696-705.

L. M. Lodish (1980). 'Applied Dynamic Pricing and Production Models with Specific Application to Broadcast Spot Pricing'. Journal of Marketing Research 17(2):203-211. 
H. Marvel \& J. Peck (1995). 'Demand uncertainty and returns policies'. International Economic Review 36(3):691-714.

T. Mazumdar, et al. (2005). 'Reference Price Research: Review and Propositions'. Journal of Marketing 69(4):84-102.

F. Miercort (1968). 'Some Effects of Advertising and Prices on Optimal Inventory Policy'. Research Report .

E. S. Mills (1959). 'Uncertainty and Price Theory'. The Quarterly Journal of Economics 73(1):116130.

E. S. Mills (1962). Price, Output, and Inventory Policy: A Study in the Economics of the Firm and Industry. Wiley.

K. Monroe (2003). Pricing: Making Profitable Decisions, third edition. McGraw-HillIrwin.

M. Nagarajan \& G. Sosic (2007). 'Stable Farsighted Coalitions in Competitive Markets'. Management Science 53(1):29-45.

M. Nagarajan \& G. Sosic (2008). 'Game-theoretic analysis of cooperation among supply chain agents: Review and extensions'. European Journal of Operational Research 187(3):719-745.

J. Nasiry \& I. Popescu (2008). 'Dynamic Pricing with Loss Averse Consumers and Peak-End Anchoring'. Working paper .

A. J. Nevins (1966). 'Some Effects of Uncertainty: Simulation of a Model of Price'. The Quarterly Journal of Economics 80(1):73-87.

N. Petruzzi \& M. Dada (1999). 'Pricing and the Newsvendor Problem: A Review with Extensions'. Operations Research 47:183-194.

N. Petruzzi \& M. Dada (2002). 'Dynamic pricing and inventory control with learning'. Naval Research Logistics 49(3):303-325.

Y. Pochet \& L. Wolsey (2006). Production Planning by Mixed Integer Programming. Springer, New York. 
L. Polatoglu (1991). 'Optimal order quantity and pricing decisions in single-period inventory systems'. International Journal of Production Economics 23(1-3):175-185.

L. Polatoglu \& I. Sahin (2000). 'Optimal procurement policies under price-dependent demand'. Int. J. Production Economics 65:141-171.

I. Popescu \& Y. Wu (2007). 'Dynamic Pricing Strategies with Reference Effects'. Operations Research 55(3):413-429.

E. Porteus (1971). 'On the Optimality of generalized $(s, S)$ policies'. Management Science 17(7):411-426.

F. Qi (2010). 'Integrating Dynamic Pricing and Replenishment Decisions Under Supply Capacity Uncertainty'. Management Science, forthcoming .

S. Ray, et al. (2005). 'Tailored Supply Chain Decision-Making Under Price-Sensitive Stochastic Demand and Delivery Uncertainty'. Management Science 51(12):1873-1891.

G. Raz \& E. L. Porteus (2006). 'A Fractiles Perspective to the Joint Price/Quantity Newsvendor Model'. Management Science 52(11):1764-1777.

H. Scarf (1960). 'The optimality of $(s, S)$ policies for the dynamic inventory problem'. Proceedings of the 1st Stanford Symposium on Mathematical Methods in the Social Sciences .

D. Simchi-Levi, et al. (2005). The Logic of Logistics: Theory, Algorithms, and Applications for Logistics Management (Second Edition). Springer, New York.

J. Song \& Z. Xue (2007). 'Demand Management and Inventory Control for Substitutable Products'. $W$ orking paper .

Y. Song, et al. (2009). 'Note: Optimal Dynamic Joint Inventory-Pricing Control for Multiplicative Demand with Fixed Order Costs and Lost Sales'. Operations Research 57(1):245-250.

S. Subrahmanyan \& R. Shoemaker (1996). 'Developing optimal pricing and inventory policies for retailers who face uncertain demand'. Journal of Retailing 72(1):7-30.

K. Talluri \& G. Van Ryzin (2004). The Theory and Practice of Revenue Management. Springer. 
S. Tayur, et al. (1998). Quantitative Models for Supply Chain Management. Kluwer Academic Publishers Norwell, MA, USA.

J. Thomas (1970). 'Price-production decisions with deterministic demand'. Management Science 16:747-750.

J. Thomas (1974). 'Price and Production Decisions with Random Demand'. Operations Research 22(3):513-518.

G. T. Thowsen (1975). 'A dynamic, nonstationary inventory problem for a price/quantity setting firm'. Naval Research Logistics Quarterly 22(3):461-476.

B. Tomlin \& Y. Wang (2008). 'Pricing and Operational Recourse in Coproduction Systems'. $M$ anagement Science 54(3):522-537.

W. van den Heuvel \& A. P. Wagelmans (2006). 'A polynomial time algorithm for a deterministic joint pricing and inventory model'. European Journal of Operational Research 170(2):463-480.

J. van Mieghem \& M. Dada (1999). 'Price Versus Production Postponement: Capacity and Competition'. Management Science 45(12):1631-1649.

H. Wagner (2004). 'Comments on "Dynamic Version of the Economic Lot Size Model"'. Management Science 50(12 Supplement):1775-1777.

H. Wagner \& T. Whitin (1958a). 'Dynamic problems in the theory of the firm'. Naval Research Logistics Quarterly 5(1):53-74.

H. Wagner \& T. Whitin (1958b). 'Dynamic Version of the Economic Lot Size Model'. Management Science 5(1):89-96.

Y. Wang (2006). 'Joint Pricing-Production Decisions in Supply Chains of Complementary Products with Uncertain Demand'. Operations Research 54(6):1110-1127.

Y. Wang, et al. (2004). 'Channel Performance Under Consignment Contract with Revenue Sharing'. Management Science 50(1):34-47.

T. Whitin (1955). 'Inventory Control and Price Theory'. Management Science 2(1):61-68. 
C. A. Yano \& S. M. Gilbert (2003). Coordinated Pricing and Production/Procurement Decisions: A Review. J. Eliashberg, A. Chakravarty, eds. Managing Business Interfaces: Marketing, Engineering, and Manufacturing Perspectives. Kluwer, Norwell, MA.

L. Yao, et al. (2006). 'Analysis of a Supply Contract for Coordinating the Newsvendor with Price Dependent Demand'. Working paper .

Q. Ye \& I. Duenyas (2007). 'Optimal Capacity Investment Decisions with Two-Sided FixedCapacity Adjustment Costs'. Operations Research 55(2):272-283.

R. Yin \& K. Rajaram (2007). 'Joint pricing and inventory control with a Markovian demand model'. European Journal of Operational Research 182(1):113-126.

L. Young (1978). 'Price, inventory and the structure of uncertain demand'. Journal of New Zealand Operations Research Society 6:157-177.

L. Young (1979). 'Uncertainty, Market Structure, and Resource Allocation'. Oxford Economic Papers 31(1):47-59.

E. Zabel (1970). 'Monopoly and Uncertainty'. The Review of Economic Studies 37(2):205-219.

E. Zabel (1972). 'Multiperiod monopoly under uncertainty'. Journal of Economic Theory 5(3):524536.

H. Zhang \& M. Fu (2005). 'Sample path derivatives for (s, S) inventory systems with price determination'. The Next Wave in Computing, Optimization, and Decision Technologies pp. 229-246.

J. Zhang \& J. Chen (2006). 'Bayesian solution to pricing and inventory control under unknown demand distribution'. Operations Research Letters 34(5):517-524.

K. Zhu \& U. Thonemann (2009). 'Coordination of pricing and inventory control across products'. $N$ aval Research Logistics 56(2):175-190.

P. Zipkin (2000). Foundations of Inventory Management. McGraw-Hill, New York. 